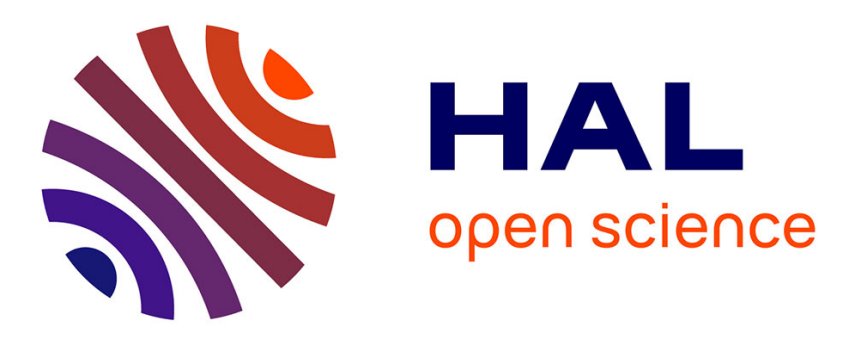

\title{
Mode selection in swirling jet experiments: A linear stability analysis
}

François Gallaire, Jean-Marc Chomaz

\section{To cite this version:}

François Gallaire, Jean-Marc Chomaz. Mode selection in swirling jet experiments: A linear stability analysis. Journal of Fluid Mechanics, 2003, november (494), pp.223-253. 10.1017/s0022112003006104. hal-01024938

\section{HAL Id: hal-01024938 \\ https://hal-polytechnique.archives-ouvertes.fr/hal-01024938}

Submitted on 27 Aug 2014

HAL is a multi-disciplinary open access archive for the deposit and dissemination of scientific research documents, whether they are published or not. The documents may come from teaching and research institutions in France or abroad, or from public or private research centers.
L'archive ouverte pluridisciplinaire HAL, est destinée au dépôt et à la diffusion de documents scientifiques de niveau recherche, publiés ou non, émanant des établissements d'enseignement et de recherche français ou étrangers, des laboratoires publics ou privés. 


\title{
Mode selection in swirling jet experiments: a linear stability analysis
}

\author{
By FRANÇOIS GALLAIRE AND JEAN-MARC CHOMAZ \\ Laboratoire d'Hydrodynamique - LadHyX, CNRS-Ecole Polytechnique, \\ F-91128 Palaiseau, France
}

(Received 28 October 2002 and in revised form 16 June 2003)

The primary goal of the study is to identify the selection mechanism responsible for the appearance of a double-helix structure in the pre-breakdown stage of so-called screened swirling jets for which the circulation vanishes away from the jet. The family of basic flows under consideration combines the azimuthal velocity profiles of Carton \& McWilliams (1989) and the axial velocity profiles of Monkewitz (1988). This model satisfactorily represents the nozzle exit velocity distributions measured in the swirling jet experiment of Billant et al. (1998). Temporal and absolute/convective instability properties are directly retrieved from numerical simulations of the linear impulse response for different swirl parameter settings. A large range of negative helical modes, winding with the basic flow, are destabilized as swirl is increased, and their characteristics for large azimuthal wavenumbers are shown to agree with the asymptotic analysis of Leibovich \& Stewartson (1983). However, the temporal study fails to yield a clear selection principle. The absolute/convective instability regions are mapped out in the plane of the external axial flow and swirl parameters. The absolutely unstable domain is enhanced by rotation and it remains open for arbitrarily large swirl. The swirling jet with zero external axial flow is found to first become absolutely unstable to a mode of azimuthal wavenumber $m=-2$, winding with the jet. It is suggested that this selection mechanism accounts for the experimental observation of a double-helix structure.

\section{Introduction}

Vortices with axial flow are encountered in many technical applications such as leading-edge vortices on delta wings or flame holders in combustion devices. Considerable effort (see Ash \& Khorrami 1995, for a complete review) has been directed towards understanding the stability characteristics of these flows since, depending both on the amount of swirl and the precise velocity profiles, they may be highly sensitive to perturbations and exhibit vortex breakdown (Leibovich 1984; Delery 1994).

The objective of the present study is to determine the temporal and absolute/convective instability properties of suitably chosen families of swirling jet/wake velocity profiles representative of real experimentally measured flow fields, such as those of Billant, Chomaz \& Huerre (1998). These basic flows differ from the well-known Batchelor profiles in two main characteristics: a decreasing circulation towards zero away from the rotating core and a smaller shear layer thickness. The predictions of the analysis are then compared to the experimentally observed characteristics of the helical structures which such flows can support. 
The swirling flow experiments in pipes of Sarpkaya (1971) and Faler \& Leibovich (1977) were crucial in the understanding of the sequence of events leading to vortex breakdown in the form of a double helix, a spiral and a bubble. In all studies, the relevant non-dimensional parameter is the swirl, which quantifies the relative importance of the swirl velocity with respect to the axial velocity. In a recent contribution, Billant et al. (1998) conducted a detailed study of the onset of vortex breakdown in a hydraulic swirling jet discharging into a large water tank at low Reynolds numbers, in the range $300<R e<1200$. As in swirling pipe flows, they determined that vortex breakdown sets in beyond a well-defined value of the swirl parameter $S \sim 1.3$, where $S$ is defined by expression (2.4). This transition is preceded by the appearance of a characteristic double-helix state of azimuthal wavenumber $m=2$ which breaks the axisymmetry of the jet. Further detailed analysis of the sequence of unsteady states supported by the jet below vortex breakdown onset was carried out by Loiseleux \& Chomaz (2003) at a Reynolds number of $R e=1490$. The double-helix state was then observed precisely at the jet outlet in the range of swirl parameters $0.6<S<1$. The aim of this study is to explain in detail the occurrence of the double helix from the linear instability analysis of the underlying swirling base flow.

There have been many attempts in the literature to relate the appearance of breakdown in swirling flows to temporal stability theory. Lessen, Singh \& Paillet (1974), Duck \& Foster (1980) and Leibovich \& Stewartson (1983) demonstrated that the swirl parameter has a plural influence on the stability of the Batchelor or $q$ vortex (see Batchelor \& Gill 1964) which satisfactorily represents trailing line vortices as well as swirling pipe flows (Faler \& Leibovich 1977; Escudier, Bornstein \& Maxworthy 1982). Negative helical modes of high azimuthal wavenumber are destabilized when the swirl is increased from zero. Further increasing the swirl above $q \sim 1.58$ completely stabilizes the Batchelor vortex. These results have been confirmed and refined in the presence of viscosity by Lessen \& Paillet (1974), Khorrami (1991) and Mayer \& Powell (1992) among others. These investigations have been somewhat inconclusive as far as the onset of vortex breakdown is concerned. Whereas the most unstable mode is found to be helical, the most robust vortex breakdown state is observed to be axisymmetric. Similarly, the occurrence of simple $(m=1)$ and double $(m=2)$ spirals cannot be accounted for by temporal stability theory since the most unstable mode corresponds to high azimuthal wavenumber $m \leqslant-4$.

More recently, temporal stability studies have been extended to account for the spatio-temporal development of disturbances. More precisely, these have sought to discriminate between convective instabilities (CI) for which perturbations are irremediably advected downstream, and absolute instabilities (AI), for which perturbations grow in the same place. In the context of vortex breakdown, this distinction appears as a natural generalization of the supercritical/subcritical flow concept introduced by Benjamin (1962). Supercritical flows allow downstream travelling waves only, whereas subcritical flows allow both upstream and downstream travelling waves. The existence of absolute instability plays a crucial role in accounting for the occurrence of synchronized self-sustained oscillations, so-called global modes, in a variety of spatially developing shear flows such as wakes, hot jets, and counterflow mixing layers as reviewed by Huerre \& Rossi (1998). It is then natural to examine whether vortex breakdown might be triggered by a transition from convective to absolute instability.

The Batchelor vortex with zero external flow was determined by Delbende, Chomaz \& Huerre (1998), Olendraru, Sellier \& Huerre (1999) and Olendraru \& Sellier (2002) 
to become absolutely or almost absolutely unstable to several helical modes of distinct symmetry $(m=-2,-3,-4, \ldots)$ at intermediate swirl settings. The analysis, however, failed to yield an unambiguous mode selection. No clear relation could be established between these spatio-temporal stability properties and axisymmetric vortex breakdown or helical structures observed in swirling jets before breakdown.

In a related study of the Rankine vortex with a top-hat jet profile (Lim \& Redekopp 1998; Loiseleux, Chomaz \& Huerre 1998), a somewhat clearer transition to absolute instability was identified via a sequence of modes $m=-2, m=-3, \ldots$ at sufficiently high swirl parameters. Loiseleux et al. (1998) accordingly suggested a criterion for vortex breakdown based on the amount of swirl necessary for the first convective/absolute transition to occur: the mode $m=-2$ is the first to undergo this transition at a swirl level $q=1.61$, which compares favourably with the experiments discussed in Spall, Gatski \& Grosch (1987). The motivation for the present study came from the fact that, though Loiseleux et al. (1998)'s study failed to account for the axisymmetric $(m=0)$ or spiral $(m=1)$ nature of vortex breakdown itself, it was nevertheless qualitatively consistent with the experimental observations of Billant et al. (1998) and Loiseleux \& Chomaz (2003) regarding the appearance of a double helix $m=2$ prior to breakdown. However, it holds for the idealized Rankine vortex with plug flow, a broken line profile which is not a proper description of the experimental swirling jet. The present study seeks to examine this issue rigorously in the context of an accurate model of the experimental velocity profiles of Billant et al. (1998). In particular, we wish to include the general effects of azimuthal shear and centrifugal instabilities, which are both absent in the case of the Batchelor vortex and the Rankine vortex with plug axial flow. It is emphasized that the study is not aimed at predicting breakdown but at explaining the origin of the dynamical state preceding breakdown.

Two methods are currently available to determine the absolute/convective nature of the instability pertaining to a given velocity profile. The classical procedure consists of applying the Briggs-Bers pinching criterion to the dispersion relation in Fourier space (Bers 1983). This method is exhaustive but it requires a subtle detailed inspection of the topology of spatial branches in the complex wavenumber plane. Alternatively, one may choose to remain in physical space and generate numerically the linear impulse response of the basic flow, as in Brancher \& Chomaz (1997) and Delbende et al. (1998). The temporal and convective/absolute instability properties are then directly retrieved from the behaviour of the impulse response wavepacket for large time. In the case of the Batchelor vortex, this procedure has been shown to yield the same results as the classical method, as demonstrated in Olendraru \& Sellier (2002).

Recent theoretical studies by Couairon \& Chomaz (1999) and Pier \& Huerre (2001), as reviewed in Huerre (2000), have reinforced the link between linear absolute/convective instability ideas and the presence of nonlinear global modes in spatially varying flows. To summarize these studies, the frequency and spatial structure of the nonlinear global mode is determined by the most upstream station to undergo an absolute instability. If the flow inlet is absolutely unstable, the global frequency is given by the real part of the absolute frequency at the inlet. If absolute instability only arises beyond a specific downstream station, the global frequency is given by the real absolute frequency at this transition station. These conclusions apply provided that the weakly non-parallel assumption holds and that linear and nonlinear absolute instability always coincide, as discussed in Chomaz (1992) and Couairon \& Chomaz (1997). In the present study, we view the pre-breakdown state as the manifestation of a nonlinear global mode within the flow. As a working 
assumption and in the absence of full knowledge of the streamwise evolution of the basic flow, we assume that it will first become absolutely unstable at the inlet. It is therefore postulated that the pre-breakdown global mode will oscillate at the absolute frequency and wavenumber prevailing at the inlet. As in the majority of realistic flows, the weakly non-parallel assumption is not rigorously valid. Secondorder effects should then be considered that leave the essential features of the global mode unchanged but significantly modify the threshold value.

The study is organized as follows. Models of the inlet swirling jet profile are discussed and compared with experiments of Billant et al. (1998) in §2. Section 3 is devoted to the non-trivial specification of the sign of variables in swirling flows. The numerical simulations of the impulse response and the diagnostic tools needed to retrieve the instability properties are outlined in $\S 4$. Section 5 pertains to the temporal instability results while $\S 6$ examines the absolute/convective instability features. The dependence of the temporal and spatio-temporal mode selection on the Reynolds number is examined in $\S 7$. A comparison with the experimentally observed characteristics of the pre-breakdown state is finally presented in $\S 8$.

\section{Swirling jet base flows}

Consider a swirling jet flow around the $x$-axis of a fluid with kinematic viscosity $v$. Let us introduce Cartesian coordinates $(x, y, z)$ as well as cylindrical coordinates $(x, r, \theta)$. In dimensional variables, indicated by an asterisk, the basic flow under consideration is

$$
\begin{gathered}
U_{x}^{*}\left(r^{*}\right)=U_{\infty}+\frac{U_{c}-U_{\infty}}{1+\left(\exp \left[\left(r^{*} / R\right)^{2} \log (2)\right]-1\right)^{N}}, \\
U_{r}^{*}\left(r^{*}\right)=0, \\
U_{\theta}^{*}\left(r^{*}\right)=\Omega_{c} r^{*} \exp \left[-\left(r^{*} / R_{v}\right)^{\alpha}\right] .
\end{gathered}
$$

In $(2.1 a), U_{\infty}$ denotes the free-stream velocity, $U_{c}$ the centreline axial velocity, and the jet core radius $R$ refers to the location of the cylindrical shear layer. The nondimensional parameter $N$ characterizes the intensity of the shear, in other words the thickness of the shear layer. The axial velocity profile $(2.1 a)$ was introduced by Monkewitz (1988) and Monkewitz \& Sohn (1988) in order to study the instabilities of circular wakes and jets. In (2.1c), $\Omega_{c}$ is the rotation rate on the axis, $R_{v}$ is a measure of the vortex core size and the non-dimensional parameter $\alpha$ represents the azimuthal shear intensity. This azimuthal velocity profile was first introduced by Carton \& McWilliams (1989) and then used by Carnevale \& Kloosterziel (1994) and Orlandi \& Carnevale (1999) to describe the plane shear instabilities of so-called isolated or screened vortices, i.e. vortices with circulation decreasing away from the core.

Using the jet core size $R$ as length scale and the centreline axial velocity defect $\Delta U \equiv U_{c}-U_{\infty}$ as velocity scale, we introduce the four non-dimensional parameters

$$
R e=\frac{\Delta U R}{v}, \quad q=\frac{\Omega_{c} R}{\Delta U}, \quad r_{v}=\frac{R_{v}}{R}, \quad a=\frac{U_{\infty}}{\Delta U} .
$$

In (2.2) Re denotes the Reynolds number, $q$ the swirl parameter which compares the intensity of the rotation to that of the advection, $r_{v}$ is the vortex to jet core radius ratio, and $a$ is the advection parameter which is a measure of the free-stream axial velocity far from the axis. An alternative would have been to consider instead parameter $\Lambda=\left(U_{c}-U_{\infty}\right) /\left(U_{c}+U_{\infty}\right)=1 /(1+2 a)$ as in Monkewitz (1988). As defined for instance in Loiseleux et al. (1998) for the Rankine vortex with plug flow, $a>0$ 

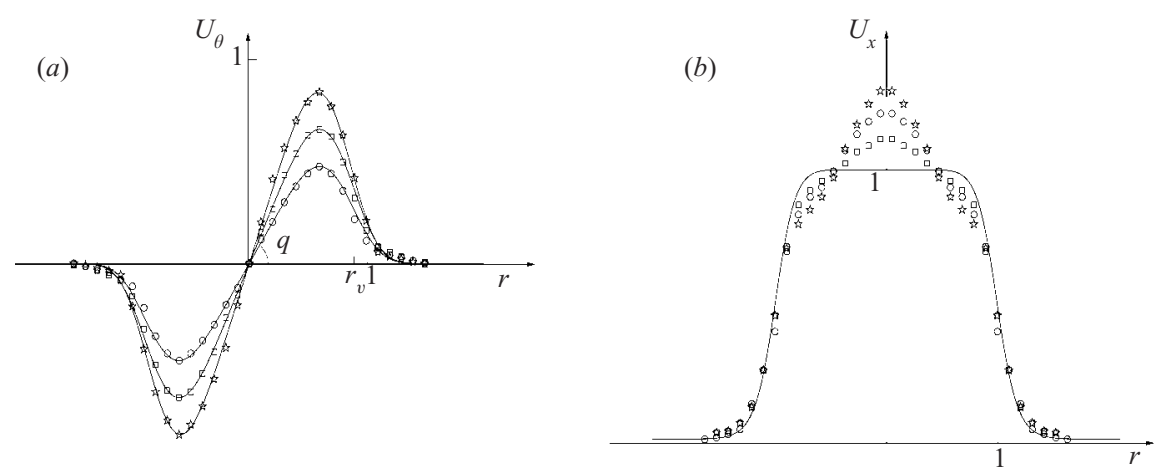

FIGURE 1. Model I basic flow field: (a) azimuthal velocity profile $(2.3 c)$ for $\alpha=4, r_{v}=0.9 ;(b)$ axial velocity profile $(2.3 a)$ for $N=3$ and $a=0$. Symbols refer to experimental data of Billant et al. (1998) at $R e=666$ and $S=0.68$ ( $\square), S=0.92(\bigcirc), S=1.08$ (汸). The correspondence between $S$ and $q$ is specified in the table of figure 2 .

corresponds to coflowing jets, $-0.5<a<0$ to counterflowing jets, $-1<a<-0.5$ to counterflowing wakes and $a<-1$ to coflowing wakes. One must emphasize that the overall direction of the axial flow changes to negative when $a \leqslant-1$. Throughout the study, the Reynolds number is set to $R e=667$, except in $\S 7$, where its influence is analysed.

In non-dimensional variables, the base flow is then

$$
\begin{gathered}
U_{x}(r)=a+\frac{1}{1+\left(\exp \left(r^{2} \log (2)\right)-1\right)^{N}}, \\
U_{r}(r)=0, \\
U_{\theta}(r)=q r \exp \left(-r / r_{v}\right)^{\alpha} .
\end{gathered}
$$

These profiles will be referred to as model I. Typical azimuthal and axial velocity distributions are displayed in figure 1, together with the experimental data obtained at $R e=666$ and for three different swirl parameters by Billant et al. (1998) and $a=0$. In order to fit the real experimental profiles of Billant et al. (1998) close to the nozzle exit, the non-dimensional parameters have been set to the values $N=3, r_{v}=0.9$ and $\alpha=4$. Note that the non-dimensional parameter $r_{v}$ which characterizes the ratio of the vortex core size to the jet core size does not coincide with any particular point in the azimuthal velocity profile and in particular not the location $r_{\max }$ of the maximum azimuthal velocity, for which $r_{\max }=r_{v}(1 / \alpha)^{(1 / \alpha)}$.

It should also be mentioned that the swirl number $q$ defined in (2.2) differs from the definition of its counterpart $S$ in Billant et al. (1998), defined by

$$
S=\frac{2 R U_{\theta}(r=R / 2)}{U_{x}(r=0)},
$$

where $R$ is the radius of the jet. The nonlinear correspondence between $q$ and $S$ is given in table 1.

Note that the experimentally measured axial velocity profile displays an overshoot near the axis, which is due to the strongly convergent nozzle of the swirling jet apparatus. This overshoot is not accounted for in the model I family of profiles, as readily seen in figure 1 . In order to evaluate its influence, we introduce another family 


\begin{tabular}{llll}
\hline & 0.68 & 0.92 & 1.08 \\
$q$ & 0.82 & 1.16 & 1.48 \\
$u_{1}$ & 0.89 & 0.83 & 0.77 \\
$u_{2}$ & 0.22 & 0.37 & 0.52 \\
$r_{c}$ & 0.42 & 0.42 & 0.42
\end{tabular}

TABLE 1. Fitting parameters of model II.

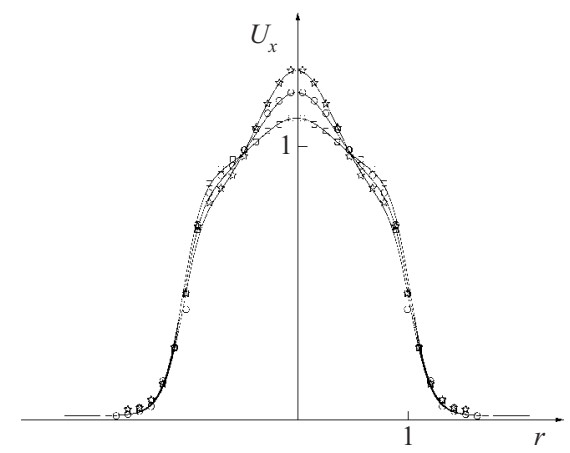

FIGURE 2. Model II axial velocity profiles given by (2.5) and accounting for the axial velocity overshoot. Symbols refer to experimental data of Billant et al. (1998) at $R e=667$ for $S=0.68$ $(\square), S=0.92(\bigcirc)$ and $S=1.08(\star)$. Parameters of model II for best fitting with experimental profiles of Billant et al. (1998) are indicated in table 1. The correspondence between $S$ and $q$ is the same as for model I.

of axial velocity profiles:

$$
U_{x}(r)=\frac{u_{1}}{1+\left(\exp r^{2} \log (2)-1\right)^{N}}+u_{2} \exp \left(-r / r_{c}\right)^{2}
$$

keeping $(2.3 b)$ and $(2.3 c)$ the same. These profiles will be referred to as model II. In (2.5), $u_{1}, u_{2}$ and $r_{c}$ depend on the swirl number $S$ and they are determined so as to provide the best possible fit with the experimental measurements, as seen in figure 2 . Corresponding best-fit values of $u_{1}, u_{2}$ and $r_{c}$ are given in table 1 . Since model I is far more generic and will be shown to capture most of the physics of screened swirling jets, the instability analysis of model II is postponed to the last section and the bulk of the study is concerned with model I. Profiles similar to model I have been measured in the swirling jet experiment of Liang (2003). Furthermore, we should draw the reader's attention to the discrepancy between the experimental measurements of Escudier et al. (1982) or Faler \& Leibovich (1977) and the fitted Batchelor profiles. In both cases, the azimuthal velocity falls off faster than $1 / r$.

\section{Sign conventions}

The literature contains contradictory sign rules. Experimentalists, for instance Escudier, Bornstein \& Zehnder (1980), choose to denote as positive the helical modes winding in the same direction as the basic flow rotation, whereas theoreticians, for instance Lessen et al. (1974), usually refer to the sign of the azimuthal wavenumber $m$ in a normal mode expansion. It is therefore appropriate to describe in some detail the conventions used in the present paper. 
Without loss of generality, only base flows with $q$ positive are considered, the negative- $q$ cases being deduced by applying the following symmetry (see Olendraru $\&$ Sellier 2002 for details):

$$
(q, m) \rightarrow(-q,-m)
$$

Swirling jets with $q \geqslant 0$ rotate in the direct sense defined by the right-hand rule with respect to the $x$-direction, i.e. the streamwise direction. In other words, the flow is rotating counter-clockwise when viewed from the downstream $x$-direction.

Infinitesimal normal mode perturbations are considered to be harmonic of the following form:

$$
u(x, r, \theta, t)=u(r) \mathrm{e}^{\mathrm{i}(k x+m \theta-\omega t)}+u^{*}(r) \mathrm{e}^{-\mathrm{i}\left(k^{*} x+m \theta-\omega^{*} t\right)},
$$

where the eigenfunction $u(r)$, the wavenumber $k$ and the frequency $\omega$ may be complex and now the asterisk stands for the complex conjugate. As a consequence, the sense of winding of a helical perturbation is given by the sign of the product $\operatorname{Re}(k) \times m$. If this product is positive, it corresponds to what we call a positive helical mode which, when travelling in the $x$-direction, winds clockwise, i.e. in opposite direction to the flow. If $R e(k) \times m$ is negative, the helical mode is negative and winds counterclockwise, i.e. in the same direction as the flow.

Expression (3.2) readily displays the real nature of the perturbation $u$, and shows that it may be equivalently represented by

$$
(k, m, \omega) \text { or }\left(-k^{*},-m,-\omega^{*}\right) .
$$

In temporal stability theory where $k$ is assumed real, the usual convention is to restrict the study to positive values of $k$, negative $k$ values being obtained through the above transformation. With this restriction, positive helical modes correspond to $m>0$ and negative helical modes to $m<0$. In that case, the sign of $\operatorname{Re}(\omega)$ gives the sign of the axial phase velocity $c=\omega / k$, and the sign of the product $m \times \operatorname{Re}(\omega)$ the sign of the azimuthal phase velocity $c_{\phi}=\omega / \mathrm{m}$.

In spatio-temporal theory, $k$ is complex and as seen later in the paper, it is convenient to let $R e(k)$ take both signs. In that instance, the winding sense of helical perturbations is determined by considering the sign of $\operatorname{Re}(k) \times m$ and not simply of $m$ as in temporal theory.

An additional complexity arises if comparison with experiments is involved. When describing vortex breakdown, one in general defines the positive streamwise direction as that of the free-stream velocity, even in the lee of the breakdown bubble where the flow is of wake type. Figure $3(a)$ depicts a typical axial velocity profile inside a breakdown bubble, as seen from an experimentalist's point of view. In the present paper as well as in many theoretical studies, the convention for the base flow is different, with the axial velocity difference $\Delta U=U_{c}-U_{\infty}$ being taken as always positive. The experimental velocity profile of figure $3(a)$ is then replaced by its symmetric counterpart in figure $3(b)$. We transfer from one to the other through an $x \rightarrow-x$ reflection symmetry and if all the experimental quantities are denoted by a prime, the transformation that should be applied is $a^{\prime}=-a, q^{\prime}=q, k^{\prime}=k, m^{\prime}=-m$ and $\omega^{\prime}=-\omega$. In that case, positive $m^{\prime}$ will correspond to negative $m$, i.e. helices rotating in the direction of swirl. 

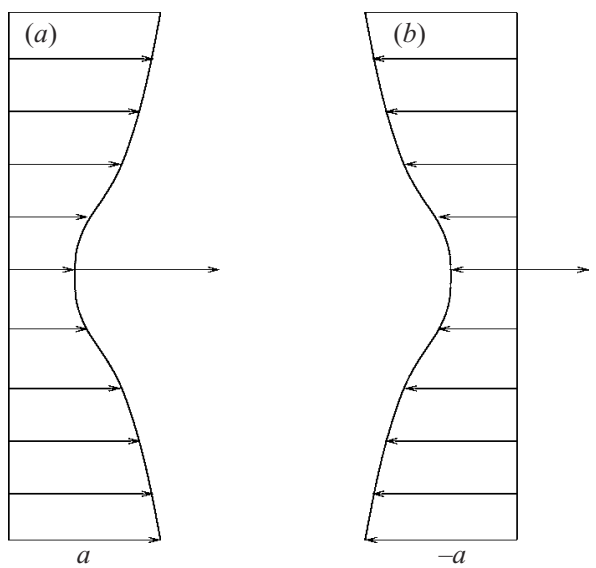

FIGURE 3. (a) Typical experimental wake profile in the lee of a breakdown bubble with a positive $x$-axis pointing downstream. $(b)$ Corresponding profile with the convention of the present paper.

\section{Numerical method and diagnostic tools}

Throughout the study, the linear temporal and spatio-temporal instability properties are retrieved from direct numerical simulations of the linear impulse response originating from an initial localized disturbance. The main feature of the method is that it gives access to the most amplified mode for each pair $k$ and $m$ of axial and azimuthal wavenumbers when temporal theory is considered, and for each pair of ray velocity $v_{g}=x / t$ and azimuthal wavenumber $m$ when spatio-temporal theory is considered. The procedure is only briefly outlined and the reader is referred to Delbende et al. (1998) for more details.

\subsection{Numerical implementation}

We consider the evolution of infinitesimal disturbances superimposed on the basic flow profiles and governed by the linearized incompressible Navier-Stokes equations. As in classical stability analysis (Drazin \& Reid 1981), the basic flow is assumed to be steady and uniform in the axial direction. Formally, this may be achieved by adding a body force that exactly compensates for the viscous diffusion of the basic flow.

We use the code described by Brancher, Chomaz \& Huerre (1994) and Delbende et al. (1998). The linearized Navier-Stokes equations are projected onto $1440 \times$ $128 \times 128$ Fourier modes in the three Cartesian directions $(O x$ is the direction of the jet axis and $O y$ and $O z$ two arbitrary perpendicular directions). In physical space, this corresponds to a cubic mesh of spacing $\mathrm{d} x=\mathrm{d} y=\mathrm{d} z=0.05$ and box lengths $L_{y}=L_{z}=6.4$ and $L_{x}=72$, the jet radius being unity as in $(2.3 a)$. The time step $\mathrm{d} t=0.005$ is chosen so as to satisfy a CFL-type numerical stability condition $\mathrm{d} x / \mathrm{d} t=10$ to be compared to a maximum velocity magnitude of order 2 . Fine resolutions in the $y$ - and $z$-directions are necessary because of the small scales of the axial and azimuthal shear layers. Such a mesh refinement has become recently available thanks to the NEC SX5 computer at IDRIS (CNRS). The sides of the box $L_{y}=L_{z}$ are chosen large enough so that confinement due to the periodicity in the $y$ - and $z$-directions is weak. The axial length $L_{x}$ of the box guarantees a high resolution $\mathrm{d} k$ in axial wavenumber while the fine mesh size $\mathrm{d} x$ gives a large cut-off wavenumber. These choices have been found to be sufficient to characterize 
properly both the temporal and the spatio-temporal instabilities. Convergence has been evaluated by performing a high-resolution simulation with $2160 \times 196 \times 196$ mesh points and $\mathrm{d} x=\mathrm{d} y=\mathrm{d} z=0.033$ for the case $q=1.2$. All the quantities to be reported in the following were found to vary by less than $1 \%$ in the temporal theory and $2 \%$ in the spatio-temporal theory. In some exceptional situations where $k=0$ modes are unstable and two unstable modes with the same azimuthal dependence have comparable amplitudes, variations were found to reach $5 \%$. This was deemed to be acceptable and results could always be improved by choosing larger integration times.

In order to mimic the Dirac delta-function forcing in space and time and to remain compatible with the cut-off wavenumber chosen here, the initial conditions are chosen as in Delbende et al. (1998). A divergence-free initial Gaussian perturbation of characteristic velocity perturbation amplitude 0.1 is contained within a sphere of radius $\rho=0.5$. The impulse is centred at $x_{0}=15, r_{0}=1$ and $\theta=15^{\circ}$ in order to feed energy into all azimuthal wavenumbers. The length scale $\rho$ is a compromise: it is small enough to reproduce a localized impulse and it respects the resolution limitations in truncated spectral space.

Once the impulse response is computed, it is analysed by choosing a state variable $a(x, y, z, t)$ which is determined at the nodes of a cylindrical grid $a(x, r, \theta, t)$. We select the axial velocity perturbation

$$
a(x, y, z, t) \equiv u_{x}(x, y, z, t) .
$$

The cylindrical coordinate space is discretized into 40 points in the radial direction in the range $0 \leqslant r \leqslant R_{\max }=L_{y}$ and 64 points in the azimuthal direction. This therefore restricts the analysed azimuthal wavenumber range to $|m|<32$, but we have systematically checked that no aliasing error is introduced by this cut-off since in all simulations modes $|m|>32$ are stable.

\subsection{Diagnostic tools}

Within a temporal framework, for a given azimuthal wavenumber $m$, the axial wavenumber $k$ is given real and the frequency $\omega_{m}(k)$ is complex and unknown. Let us introduce the azimuthal Fourier transform $a(x, r, m, t)$ of $a(x, r, \theta, t)$ as well as the double (axial and azimuthal) Fourier transform $a(k, r, m, t)$. For a given wavenumber pair $(k, m)$, the behaviour of $a(k, r, m, t)$ is dominated for large time by the most unstable mode of eigenfunction $f(r)$ and complex frequency $\omega_{m}(k)$, according to the relation

$$
a(k, r, m, t) \propto f(r) \mathrm{e}^{-\mathrm{i} \omega_{m}(k) t}, \quad t \rightarrow \infty .
$$

The definitions and discrete-time approximations of the growth rate $\omega_{m, i}(k)$ and the frequency $\omega_{m, r}(k)$ are listed in table 2 .

As noticed by Delbende et al. (1998), the times $t_{1}$ and $t_{2}$ and the time-increment $t_{2}-t_{1}$ used to numerically evaluate these quantities have to be large enough to ensure convergence on the leading most unstable mode and circumvent low-frequency oscillations. In order to guarantee that the transient regime has ended, we have chosen $t_{1}$ so that $A_{m}\left(k, t_{1}\right)$ is at least three orders of magnitude larger than the initial amplitude $A_{m}(k, 0)$. All growth rates have been computed by setting $t_{2}=2 t_{1}$. In addition we have checked that the growth rate computed for two other time intervals $\left(t_{1}, 1.5 t_{1}\right)$ and $\left(1.5 t_{1}, 2 t_{1}\right)$ differs by less than $1 \%$ from the value obtained with the nominal range $\left(t_{1}, 2 t_{1}\right)$. By contrast, in order to compute the mode frequency, the time interval $t_{3}-t_{2}$ must be short enough (Delbende et al. 1998) to circumvent the difficulties associated 
Nature

Signal

Amplitude

Phase

Growth rate

Numerical approximation

Frequency

Numerical approximation
Definition

$$
\begin{aligned}
& a(k, r, m, t)=\int_{-\infty}^{+\infty} \int_{0}^{2 \pi} a(x, r, \theta, t) \mathrm{e}^{-\mathrm{i}(k x+m \theta)} \mathrm{d} x \mathrm{~d} \theta \\
& A_{m}(k, t)=\left(\int_{0}^{R_{t o t}}|a(k, r, m, t)|^{2} r \mathrm{~d} r\right)^{1 / 2} \\
& \phi_{m}(k, t)=\arg \left(a\left(k, r_{0}, m, t\right)\right), \text { with } 0 \leqslant \phi_{m}(k, t)<2 \pi
\end{aligned}
$$$$
\omega_{m, i}(k)=\lim _{t \rightarrow \infty} \frac{\partial}{\partial t} \ln A_{m}(k, t)
$$$$
\omega_{m, i}(k) \sim \frac{\ln \left(A_{m}\left(k, t_{2}\right) / A_{m}\left(k, t_{1}\right)\right)}{t_{2}-t_{1}}
$$$$
\omega_{m, r}(k)=\lim _{t \rightarrow \infty}-\frac{\partial}{\partial t} \phi_{m}(k, t)
$$$$
\omega_{m, r}(k) \sim-\frac{\phi_{m}\left(k, t_{3}\right)-\phi_{m}\left(k, t_{2}\right)}{t_{3}-t_{2}}
$$

TABLE 2. Definitions and approximations of the different characteristic quantities used in temporal stability theory.

with the discontinuous nature of the phase function $\phi_{m}(k, t)$ whenever it reaches 0 or $2 \pi$ (see table 2 ).

In the spatio-temporal formulation, the development of the wavepacket along rays of specific velocity $x / t=v_{g}$ is considered.

According to steepest descent arguments (Bers 1983), the long-time behaviour of the impulse response along each spatio-temporal ray $x / t=v_{g}$ is dominated by the mode with highest spatio-temporal growth rate according to

$$
a(x, r, m, t) \propto g(r) t^{-1 / 2} \exp \left[k_{m}\left(v_{g}\right)\left(x-x_{0}\right)-\omega_{m}\left(v_{g}\right) t\right], t \rightarrow \infty,
$$

where $g(r)$ is the eigenfunction and $k_{m}\left(v_{g}\right)$ and $\omega_{m}\left(v_{g}\right)$ represent the complex wavenumber and frequency travelling at the group velocity $v_{g}$. In (4.3), the real part of the exponent

$$
\sigma_{m}\left(v_{g}\right)=\omega_{m, i}\left(v_{g}\right)-v_{g} k_{m, i}\left(v_{g}\right)
$$

denotes the temporal growth rate observed when travelling at the group velocity $v_{g}$.

The definitions and discrete-time approximations pertaining to the growth rate and to the imaginary and real parts of the complex wavenumber and the local complex frequency are listed in table 3. We emphasize, for reasons to be explained below, that we have not used a demodulation procedure based on the Hilbert transform as in Delbende et al. (1998). According to signal processing theory (Schwartz, Bennett \& Stein 1966), the Hilbert transform degrades the tails of the wavepacket considerably when its spectrum does not vanish at $k=0$. The accurate determination of the leadingand trailing-edge velocities of the wavepacket then becomes hazardous. In such cases, the edges of the wavepacket are still accessible by making use of the usual azimuthal Fourier transform $a(x, r, m, t)$ of the real signal $a(x, r, \theta, t)$. This procedure, however, has a shortcoming: the phase and amplitude variations along a ray are less precisely distinguished, resulting in a decrease in accuracy. This remains acceptable if larger times $t_{1}$ and $t_{2}$ are used. Note that positive and negative helical modes cannot be separated and the mode with the largest growth rate will dominate. This feature can 
Nature

Signal

Amplitude

Phase

Growth rate

Numerical approximation

Frequency

Numerical approximation

Wavenumber

Numerical approximation

$k_{m, i}$

Numerical approximation

$\omega_{m, i}$

TABLE 3. Definitions and app $a(x, r, m, t)$

$A_{m}(x, t)=\left(\int_{0}^{R_{t o t}}|a(x, r, m, t)|^{2} r \mathrm{~d} r\right)^{1 / 2}$

$\phi_{m}(x, t)=\arg \left(a\left(x, r_{0}, m, t\right)\right)$, with $0 \leqslant \phi_{m}(k, t)<2 \pi$

$\sigma_{m}\left(v_{g}\right)=\lim _{t \rightarrow \infty} \frac{\partial}{\partial t} \ln \left(t^{1 / 2} A_{m}\left(v_{g} t+x_{0}, t\right)\right)$

$\sigma_{m}\left(v_{g}\right) \sim \frac{\ln \left(A_{m}\left(x_{0}+v_{g} t_{2}, t_{2}\right) / A_{m}\left(x_{0}+v_{g} t_{1}, t_{1}\right)\right)}{t_{2}-t_{1}}+\frac{\ln \left(t_{2}-t_{1}\right)}{2\left(t_{2}-t_{1}\right)}$

$\omega_{m, r}\left(v_{g}\right)=\lim _{t \rightarrow \infty}-\frac{\partial}{\partial t} \phi_{m}\left(v_{g} t, t\right)$

$\omega_{m, r}\left(v_{g}\right) \sim-\frac{\phi_{m}\left(x_{0}+v_{g} t_{3}, t_{3}\right)-\phi_{m}\left(x_{0}+v_{g} t_{2}, t_{2}\right)}{t_{3}-t_{2}}$

$k_{m, r}\left(v_{g}\right)=\lim _{t \rightarrow \infty} \frac{\partial}{\partial x} \phi_{m}\left(v_{g} t, t\right)$

$k_{m, r}\left(v_{g}\right) \sim \frac{\phi_{m}\left(x_{0}+v_{g} t_{2}+\delta x, t_{2}\right)-\phi_{m}\left(x_{0}+v_{g} t_{2}, t_{2}\right)}{\delta x}$

$k_{m, i}\left(v_{g}\right)=-\frac{d}{d v_{g}} \sigma_{m}\left(v_{g}\right)$

$k_{m, i}\left(v_{g}\right) \sim-\frac{\sigma_{m}\left(v_{g}+\delta x / t_{2}\right)-\sigma_{m}\left(v_{g}\right)}{\delta x / t_{2}}$

$\omega_{m, i}=\sigma_{m}\left(v_{g}\right)+v_{g} k_{m, i}\left(v_{g}\right)$

approximations of different characteristic quantities used in spatio-temporal stability theory

be seen by the fact that, due to the real nature of $a(x, r, \theta, t)$,

$$
a(x, r, m, t)=a^{*}(x, r,-m, t) .
$$

As a consequence, the convention adopted in temporal stability theory cannot be pursued: the winding direction of a helical mode on a given ray $x / t=v_{g}$ is not given by the sign of $m$ but by the sign of the product $m \times k_{m, r}$. Furthermore, since the signal is not demodulated, growth rates and other quantities may oscillate in time and space due to the phase variations. This phase jitter is only significant on rays where the two symmetric positive and negative helical modes are of equal amplitude. In our study, as will become clear in $\S 5$, the negative helical modes are in general considerably more unstable than their positive counterparts, resulting in a satisfactory determination of the mode characteristics in all cases of interest.

\section{Temporal instability}

It is well known (Delbende et al. 1998) that temporal stability properties depend on the advection parameter $a$ through a trivial Doppler shift. Throughout this section, we therefore assume $a=0$ without loss of generality. 


\subsection{Inviscid instablity mechanisms and general criteria}

The models under consideration are in many respects distinct from the classical Batchelor or $q$-vortex which is often used to describe fully developed trailing line vortices.

When $q$ is set to zero, one recovers a cylindrical jet or wake profile in the absence of rotation. Flows with axial shear have been studied by Rayleigh (1892) and Batchelor \& Gill (1964) in order to generalize the well-known inflection point criterion. For a given axial wavenumber $k$ and azimuthal wavenumber $m$ a necessary condition for inviscid instability is that the quantity $\left(r U_{x}^{\prime}\right) /\left(m^{2}+k^{2} r^{2}\right)$ displays a local extremum somewhere in the fluid. As noticed by Batchelor \& Gill (1964) or Lessen \& Singh (1974), this condition is in general too weak: for instance the fully developed jet is unstable only to the $m=1$ mode, whereas the necessary condition is satisfied for all $m$.

When $q$ goes to infinity, the axial velocity becomes negligible and one recovers a pure vortex with a screened azimuthal velocity profile $u_{\theta}(r)$. Such a screened (or isolated) vortex will be unstable to two-dimensional $(k=0)$ as well as axisymmetric $(m=0)$ and helical disturbances. This property makes it quite distinct from the Lamb-Oseen (also referred to as Burgers) vortex which remains neutrally stable in the inviscid limit. Note also that the Lamb-Oseen azimuthal velocity profile is the same as for the Batchelor vortex. According to the generalization of the two-dimensional inflection point criterion to a circular geometry (see Rayleigh 1892; Drazin \& Reid 1981), the existence of a local extremum in the axial vorticity associated with $U_{\theta}(r)$ constitutes a necessary condition for instability to two-dimensional disturbances of the form $\mathrm{e}^{\mathrm{i} m \theta}$. Thus, the presence of azimuthal shear is very likely to destabilize the screened vortex. But this is not the only potentially destabilizing agent: by virtue of the sufficient (Rayleigh 1916) and necessary (Synge 1933) Rayleigh criterion, an axisymmetric rotational flow $U_{\theta}(r)$ is unstable to axisymmetric perturbations if and only if the square of its circulation decreases in the radial direction. Centrifugal instability will therefore also affect the screened vortex.

When $q$ is finite but non-zero, the physical effects described above all come into play. Several inviscid criteria have been established: the centrifugal instability Rayleigh criterion has been generalized by Howard \& Gupta (1962) to vortices with axial flow, i.e. swirling jets. In contrast to the case of a pure vortex, it only gives a necessary condition for instability to axisymmetric modes $m=0$. A local condition for helical instability has been proposed by Ludwieg (1961), under the assumption of a narrow annulus of fluid. Leibovich \& Stewartson (1983) later derived a more stringent sufficient (but not necessary) condition for helicoidal instability which is further discussed in $\S 5.6$.

It may easily be verified that these inviscid criteria are all fulfilled by the base flow (2.3), but none predicts which mode will dominate. The objective of this paper is to determine precisely the spatio-temporal structure of the dominant mode, in particular the selected wavenumber pair $(k, m)$.

\subsection{Non-swirling jets}

Figure 4(a) presents the growth rate $\omega_{m, i}(k)$ of the unstable modes as a function of the axial wavenumber $k$. Note that in the absence of rotation, the positive and negative helical modes have the same growth rate. Only $m=0, m= \pm 1, m= \pm 2$ and $m= \pm 3$ are unstable, higher-order modes being stable for all $k$. The overall highest growth rate is reached at $k=1.8$ for the bending modes $m= \pm 1$. Although there exist spatiotemporal results on the family of axial velocity profiles introduced by Monkewitz 

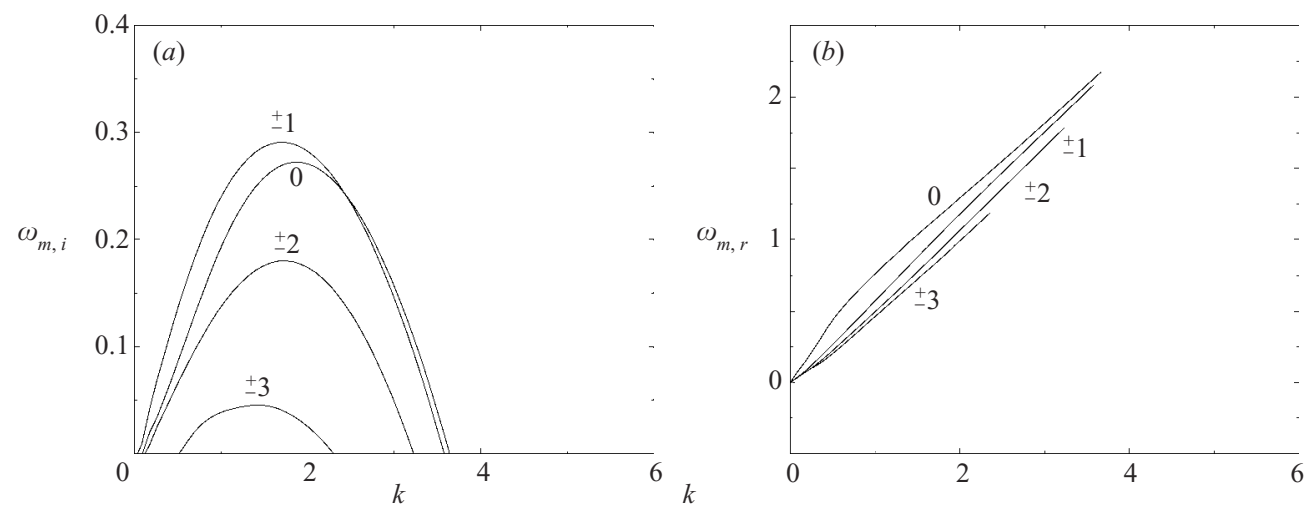

FiguRE 4. Model I temporal instability of a pure jet, i.e. at $q=0 .(a)$ growth rate $\omega_{m, i}(k)$ as: a function of axial wavenumber $k ;(b)$ frequency $\omega_{m, r}(k)$ as a function of $k$. Only the unstable portion of $\omega_{m, r}$ is displayed.

(1988) and used in this study, there are no corresponding viscous or inviscid temporal stability results. Still, a qualitative comparison may be made with the spatial stability analysis of Michalke (1984) for the model profile $U_{x}(r)=\frac{1}{2}[1+\tanh ((1 / r-r) / 4 \Theta)]$, where $\Theta$ is the non-dimensional shear layer thickness, provided that both the present profile and Michalke's correspond to the same axial shear layer thickness

$$
\Theta=\int_{0}^{\infty} U_{x}(r)\left[1-U_{x}(r)\right] \mathrm{d} r .
$$

Michalke (1984) determined that the most unstable mode is $m=1$ when $\Theta \geqslant 1 / 5$ and $m=0$ when $\Theta \leqslant 1 / 10$. For $0.1 \leqslant \Theta \leqslant 0.2, m=1$ and $m=0$ are in close competition. For the axial velocity profile $(2.3 a)$, the shear layer thickness ratio $\Theta$ equals 0.13 at $N=3$ and figure $4(a)$ indicates that the most unstable mode is $m= \pm 1$ with $m=0$ having a comparable but less intense growth rate. The present results are therefore consistent with Michalke's analysis.

Figure $4(b)$ represents the associated real part of the frequency $\omega_{m, r}$. It is seen that, as a first approximation, the phase velocity $\omega_{m, r} / k$ remains equal to 0.5 , the average velocity between the free stream $a=0$ and the jet velocity of unity.

\subsection{Swirling jets of moderate swirl, $q=0.4$}

As soon as a small amount of swirl is applied, negative azimuthal modes become unstable. For instance, figure 5(a), which is is the analogue of figure 4(a) but for a swirl parameter $q=0.4$, shows strong destabilization of negative azimuthal wavenumbers up to $m=-14$. The overall maximum growth rate is attained for $m=-1$. It may be seen that $m= \pm 2$ and $m= \pm 3$ are unstable at $k=0$, i.e. for pure two-dimensional perturbations in a cross-axial plane. Modes $m=2$ and $m=-2$, resp. $m=3$ and $m=-3$, have the same non-zero growth rate at $k=0$. When $k=0$ the only destabilizing mechanism is the azimuthal shear, the axial velocity field losing all its influence on the development of the instabilities. For an azimuthal shear parameter $\alpha=4$, Carton \& McWilliams (1989), Carnevale \& Kloosterziel (1994) and Orlandi \& Carnevale (1999) have shown that the only unstable modes of an isolated vortex with azimuthal velocity profile $(2.1 c)$ and $\Omega_{c}=1$ and no axial flow are $m=2$ and $m=3$ with inviscid growth rates $\omega_{2, i}(0)=0.1$ and $\omega_{3, i}(0)=0.05$, in reasonable agreement with the present 

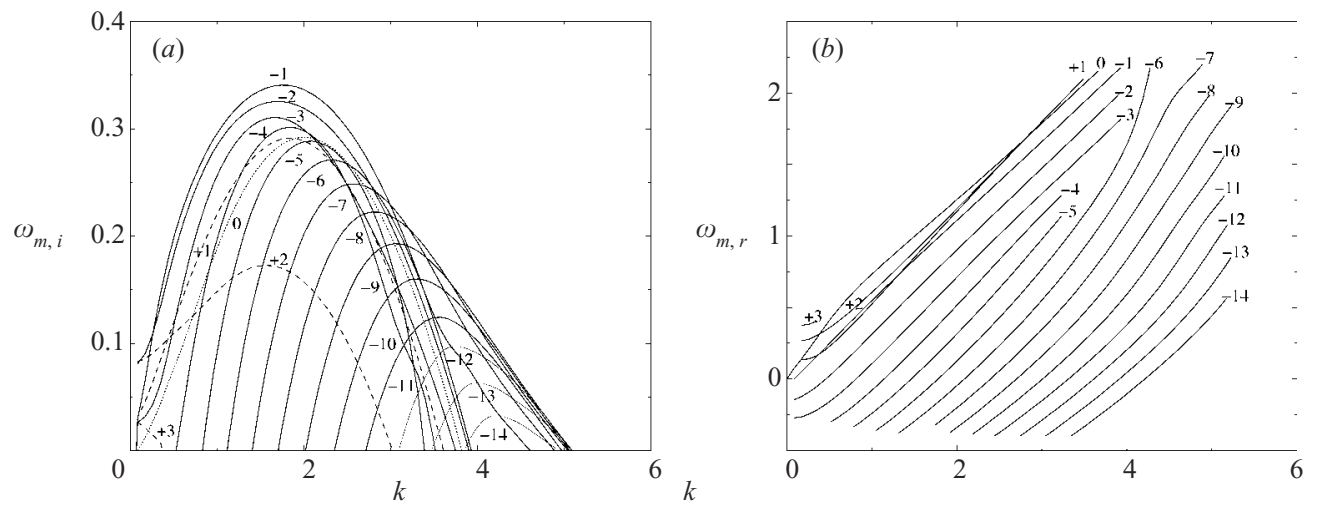

FIgURE 5. Model I temporal instability at $q=0.4$ for moderate swirl: $(a)$ growth rate $\omega_{m, i}(k)$ as a function of axial wavenumber $k ;(b)$ frequency $\omega_{m, r}(k)$ as a function of $k$.
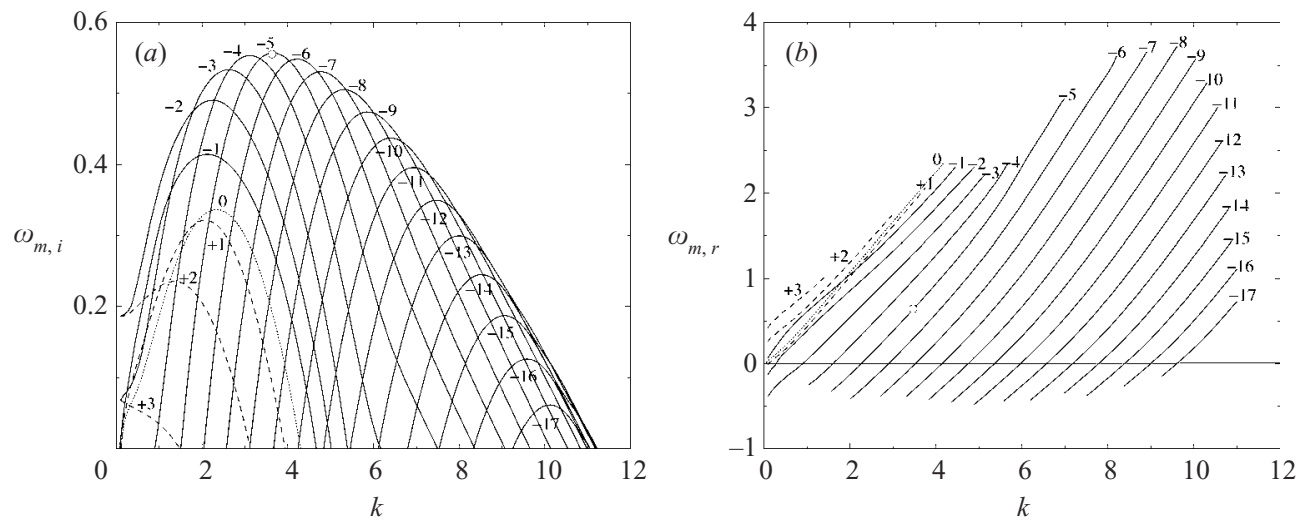

FIGURE 6. Model I temporal instability at $q=0.8:(a)$ growth rate $\omega_{m, i}(k)$ as a function of axial wavenumber $k ;(b)$ frequency $\omega_{m, r}(k)$ as a function of $k$. These stability diagrams are representative of swirl values $q \geqslant 0.8$.

viscous results. The precise evolution of the instability modes from the non-rotating case $(\S 5.2)$ to the moderately rotating case will be detailed in $\S 5.5$.

\subsection{Swirling jets with large swirl values $q=0.8$ and $q=1.2$}

For larger swirl, $m=-1$ is no longer the leading mode but is replaced by $m=-5$. Typical instability curves are displayed in figure 6 , for swirl $q=0.8$. As in the previous case, $m= \pm 2$ and $m= \pm 3$ are unstable at $k=0$, with the same growth rate for positive and negative modes.

As $k$ increases from zero, in addition to the $m= \pm 2$ and $m= \pm 3$ modes, the bending $m=+1$ and $m=-1$ as well as the axisymmetric $m=0$ modes become unstable followed by the negative helical modes $m=-4$ to $m=-17$. Each mode $m$ is unstable in a finite wavenumber range $\left[k_{m}^{a} ; k_{m}^{c}\right]$ with for example $k_{m}^{a}=0$ for $-3 \leqslant m \leqslant 3$. Note that both $k_{m}^{a}$ and $k_{m}^{c}$ are monotically decreasing functions of $-16 \leqslant m \leqslant 3$. Each mode reaches its maximum growth rate $\omega_{m, i}^{\max }$ at $k_{m}^{\max }$. The value of $\omega_{m, i}^{\max }$ first increases with $m$ and reaches its overall maximum for $m=-5$, indicated by a circle at $k_{-5}^{\max }=3.8$ in figure 6 , before decreasing continuously. 

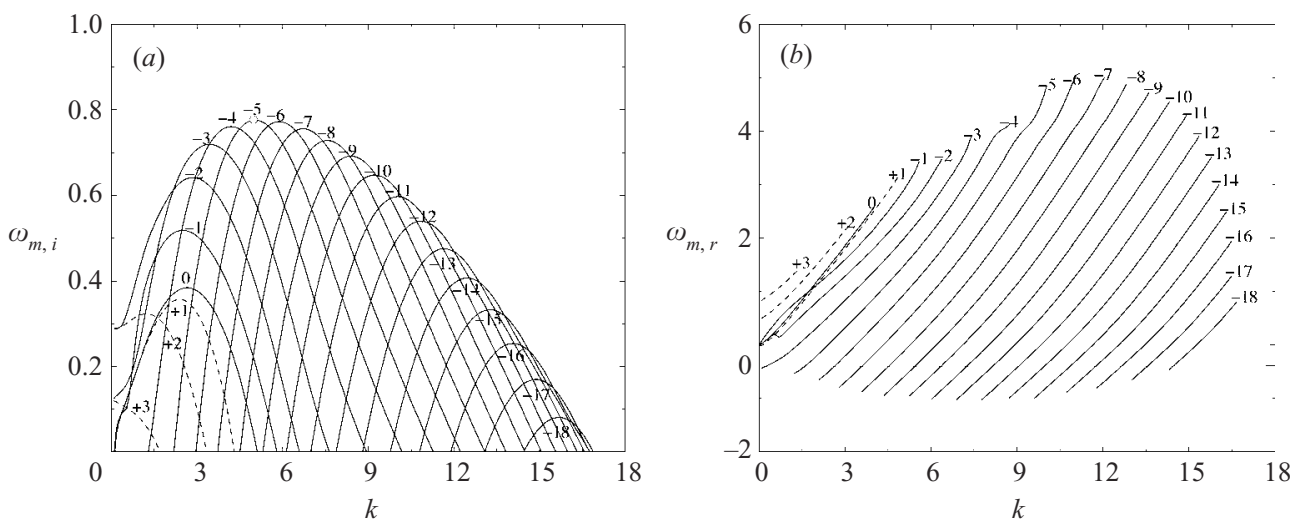

FIGURE 7. Model I temporal instability at $q=1.2:(a)$ growth rate $\omega_{m, i}(k)$ as a function of axial wavenumber $k ;(b)$ frequency $\omega_{m, r}(k)$ as a function of $k$. These stability diagrams are representative of swirl values $q \geqslant 0.8$.

Figure 6(b) depicts the corresponding frequency $\omega_{m, r}(k)$ as a function of $k$ for the unstable azimuthal modes in their unstable wavenumber range. Positive helical modes $m \geqslant 0$ are seen to possess positive frequencies indicating that they rotate in time in the same direction as the flow. Negative helical modes $m<-1$ have negative frequencies at small $k$ and positive frequencies at large $k$, indicating that they rotate in time in the same direction as the flow at small $k$ but in the opposite direction to the flow at large $k$. At the overall maximum growth rate for $k_{-5}^{\max }=3.6$, the negative helical mode $m=-5$ is seen to rotate in time in the opposite direction to the vortex. The bending mode $m=-1$ is quite peculiar since it rotates in the opposite direction to the swirling jet in the whole unstable wavenumber range.

The discussion concerning $q=0.8$ is in fact much more general. For $q \geqslant 0.8$, we have observed that the temporal stability results are very similar to the $q=0.8$ case, except that all growth rates increase linearly with $q$. The similarity between figure 6 at $q=0.8$ and figure 7 at $q=1.2$ provides an illustrative example. In the large- $q$ limit, the flow becomes analogous to a pure vortex without axial flow and at infinite Reynolds number. The only existing studies on the stability of pure isolated vortices are either a full three-dimensional stability study at $R e=667$ (Gallaire \& Chomaz 2003 ), or an inviscid study limited to the two-dimensional limit $k=0$ (Carnevale \& Kloosterziel 1994), or restricted to $\alpha=2$ and $|m| \leqslant 2$ (Gent \& McWilliams 1986). In view of these analyses, it is expected that the flow remains unstable for the modes $m=0, m= \pm 1, m= \pm 2$ and $m= \pm 3$ as $q$ continues to increase, in strong contrast to the Batchelor vortex.

\subsection{Dependence on swirl parameter}

In order to analyse in detail the evolution of the growth rates as the swirl is increased, we depict in figure 8 contours of the growth rates in the $(k, q)$-plane for different azimuthal wavenumbers. The $m=0$ bulging mode (figure $8 a$ ) is unstable without rotation, the axial shear leading to the formation of vortex rings via the classical jet instability. As $q$ is increased, the growth rate of all wavenumbers increases (figure $8 a$ ). The centrifugal instability cooperates with the axial shear to enhance the instability of the axisymmetric mode. Larger axial wavenumbers are then destabilized. The growth 

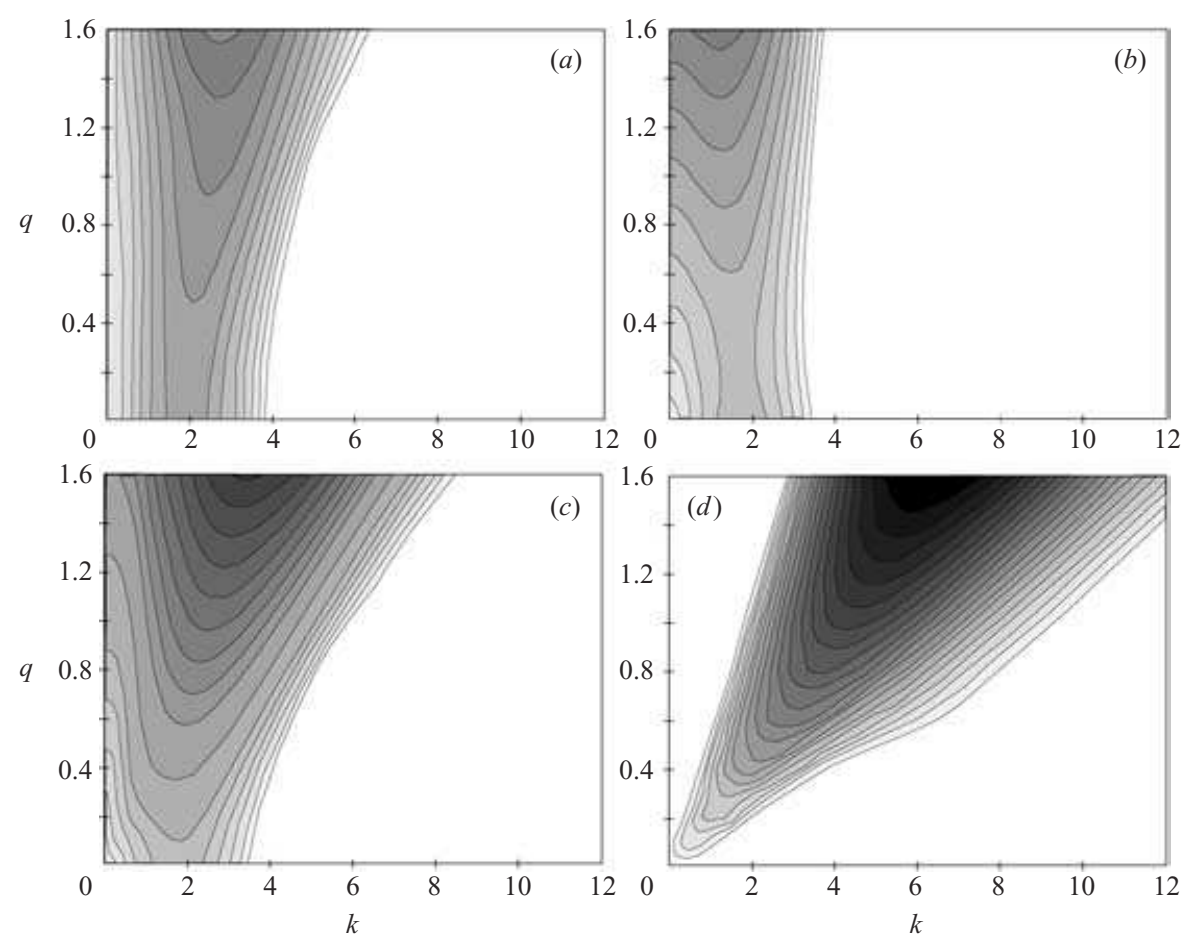

FIGURE 8. Model I contours of the growth rate for representative modes in the $(k, q)$-plane. The interval between two neighbouring contours is $\mathrm{d} \sigma=0.05$ and the grey scale is the same on all four plots. (a) $m=0 ;(b) m=+2 ;(c) m=-2$ and $(d) m=-5$. The white zone corresponds to negative values of the growth rate (stable region).

rate at small $k$ is insensitive to $q$ since the instability is only due to the axial shear at large wavelength. The maximum growth rate increases with $q$ and it is reached for a slowly increasing value of the axial wavenumber close to $k=2$.

For $m=+2$, the overall behaviour is reversed (figure $8 b$ ): small axial wavenumbers are strongly destabilized by the presence of rotation and even $k=0$ becomes unstable. This corresponds to the effect of the azimuthal shear that renders the two-dimensional flow in the $(r, \theta)$-plane unstable. By contrast, axial wavenumbers beyond $k=2$ are almost unaffected by the rotation. At small $k$, the negative mode $m=-2$ (figure $8 c$ ) exhibits a behaviour remarkably similar to $m=+2$ (figure $8 b$ ) since both modes are controlled by the azimuthal shear instability for such large wavelengths. For axial wavenumbers larger than $k=2$, the mode $m=-2$ is strongly destabilized by the rotation. This feature indicates that, at large $k$, the centrifugal effect is stabilizing for $m=+2$ but destabilizing for $m=-2$. The explanation for the strong difference between the stability properties of positive and negative helical modes constitutes the topic of the next subsection. For $m=-5$ (figure $8 d$ ) swirl strongly promotes the instability and the unstable $k$-range drifts towards large $k$ values.

\subsection{Comparison with the centrifugal instability analysis of Leibovich \& Stewartson (1983)}

The strong destabilization of the negative helical modes observed for instance in the range $q \geqslant 0.8$ but already apparent for $q=0.4$, is reminiscent of the results obtained 
by Mayer \& Powell (1992) and Khorrami (1991), among others, for the Batchelor vortex, in spite of the strong differences in the profile shapes and in the resulting active instability mechanisms. The main difference is that, when inviscid instability modes are concerned, as the swirl ratio becomes larger than a finite value of $q(q=1.58$ in the inviscid limit), the Batchelor vortex restabilizes whereas the present swirling jet flow does not.

The underlying physical reason for the dominance of negative helical modes as a general feature of swirling jets may be found in the early work of Ludwieg (1960), in the short note of Emanuel (1984) or in the detailed large- $m$ asymptotic study of Leibovich \& Stewartson (1983). Its most likely interpretation as a manifestation of centrifugal instability in a sloped reference frame aligned with the direction of vanishing strain as outlined below.

According to Leibovich \& Stewartson (1983), at any given radius $r_{0}$, it is possible to construct an eigenmode centred on $r_{0}$ with a maximum growth rate, at leading order in $(1 / m)^{-1 / 2}$, reached for the $(k, m)$ wavenumber pairs such that

$$
\beta=-k / m=\left.\frac{\mathrm{D}\left(U_{\theta} / r\right)}{\mathrm{D} U_{x}}\right|_{r_{0}},
$$

where $\mathrm{D}$ stands for $\mathrm{d} / \mathrm{d} r$ and $\beta=-k / m$ is the helix angle.

Stewartson \& Leibovich (1987) emphasize that the direction defined by the third term in (5.2) coincides with the direction of zero rate-of-strain vector. Hence, expression (5.2) states that the most unstable wavenumber pairs $(k, m)$ are aligned along the zero rate-of-strain direction.

At any radius $r_{0}$, it is then possible to let $k$ and $m$ both go to infinity with $\beta=-\mathrm{k} / \mathrm{m}$ fixed and determined by (5.2). According to Leibovich \& Stewartson (1983), the growth rate $\sigma\left(r_{0}\right)$ then tends to

$$
\sigma^{2}\left(r_{0}\right)=\left.\frac{2 U_{\theta}\left(r \mathrm{D} U_{\theta}-U_{\theta}\right)\left(U_{\theta}^{2} / r^{2}-\left(\mathrm{D} U_{\theta}\right)^{2}-\left(\mathrm{D} U_{x}\right)^{2}\right)}{\left(r \mathrm{D} U_{\theta}-U_{\theta}\right)^{2}+r^{2}\left(\mathrm{D} U_{x}\right)^{2}}\right|_{r_{0}}
$$

This function reaches a maximum $\sigma_{\max }$ at $r_{0}=r_{c}$, thereby corresponding to a helix angle $\beta$ of the wavevector given by (5.2) evaluated at $r_{c}$. Figure 9 depicts the evolution of $\sigma_{\max }(q), r_{c}(q)$ and $\beta(q)$ for the base flow (2.3) and for the Batchelor vortex. As shown by Leibovich \& Stewartson (1983) and readily seen in figure 9, this centrifugal instability mechanism is not active once $q>\sqrt{2}$ for the Batchelor vortex since $r_{c}$ and $\sigma_{\max }$ both vanish for $q=\sqrt{2}$. By contrast, for the swirling jet under consideration, it is seen that the growth rate increases linearly with the swirl $q$. The most unstable helix angle increases gradually whereas the localization of the eigenmodes $r_{c}(q)$ barely changes.

One should note that the characteristics of this generalized centrifugal instability of Leibovich \& Stewartson (1983) and Ludwieg (1960) are qualitatively and even quantitatively similar at moderate swirl for both models. Negative large values of the azimuthal wavenumber are therefore destabilized by the generalized centrifugal instability and are relatively insensitive to the details of the velocity profiles. This generality is remarkable and explains why the Batchelor vortex is able to capture instability features of many low-swirl flows. At larger swirl values, the temporal instability properties become specific to each profile.

In order to verify that the inviscid mechanism of Leibovich \& Stewartson (1983) drives the instability of model I, we have represented in figure 10 the computed growth rate $\omega_{m, i}$ for $q=1.2$ and all $m \leqslant-2$ as a function of the helix angle $\beta=-k / m$, instead 


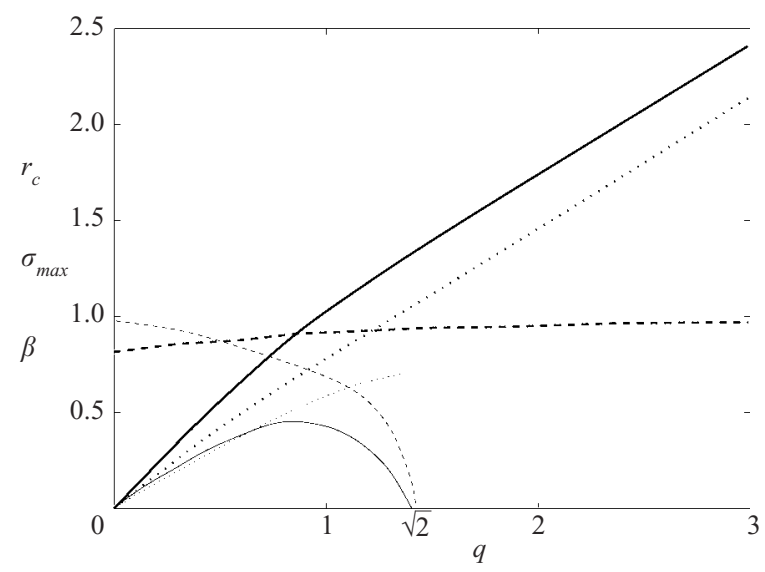

FIGURE 9. Influence of the swirl parameter $q$ on the maximum asymptotic growth rate $\sigma_{\max }$ predicted by Leibovich \& Stewartson (1983) (solid line), the corresponding radius $r_{c}$ (dashed line) and the most unstable helix angle $\beta$ (dotted line) respectively for the model I screened swirling jet (heavy lines) and the Batchelor vortex (thin lines).

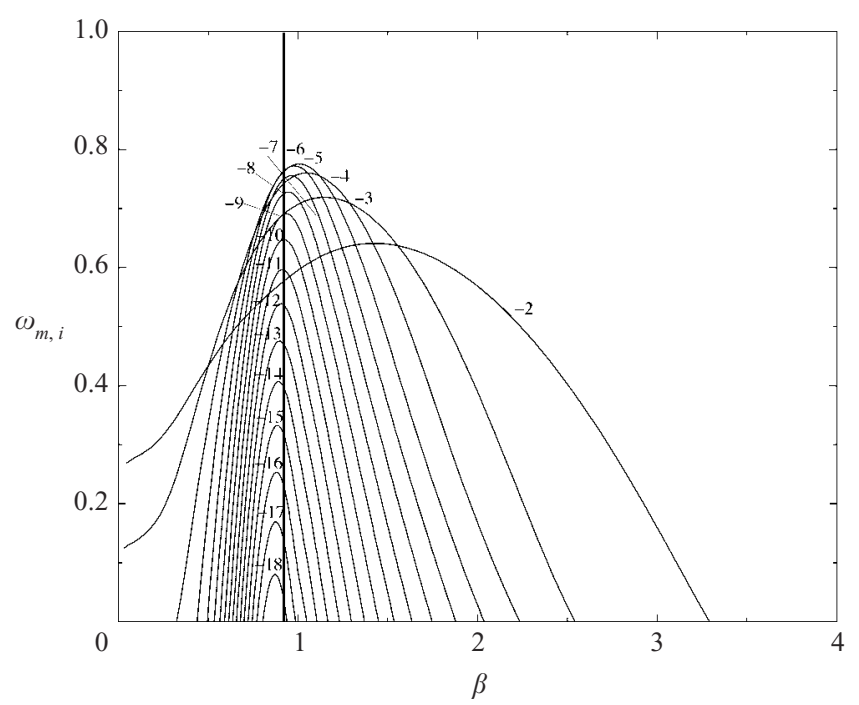

FIGURE 10. Model I growth rate $\omega_{m, i}$ as a function of helix angle $\beta=-k / m$ for $q=1.2$ and various values of $m$ in the range $-18 \leqslant m \leqslant-2$.

of $k$ as in figure 7. All curves from $m=-5$ to $m=-18$ then reach a maximum for the same value of $\beta$ close to 0.92 , as predicted by the Leibovich \& Stewartson (1983) asymptotic theory.

Since the computed growth rates are affected by viscosity, modes with large wavenumbers are seen to be quenched, in contrast with the inviscid asymptotic theory. Nonetheless, the maximum growth rate for $m=-5$ and $\beta$ near $\beta_{\max }=0.92$ is close to 0.78 , which compares favourably with the predicted inviscid value of 1.1 . The analysis of the influence of viscosity is postponed to $\S 7$. 


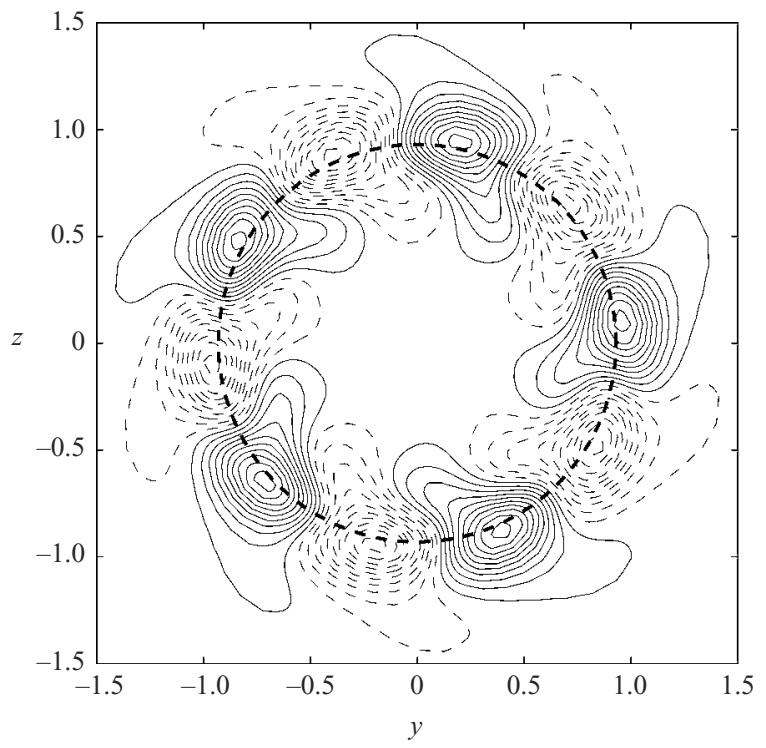

FIGURE 11. Isocontours of the model I axial velocity eigenfunction in the transverse $(y, z)$-plane for the $m=-5$ mode of maximum growth rate at $k_{-5}^{\max }=4.9 ; q=1.2$. The dashed circle represents the characteristic radius predicted by Leibovich \& Stewartson's asymptotic theory.

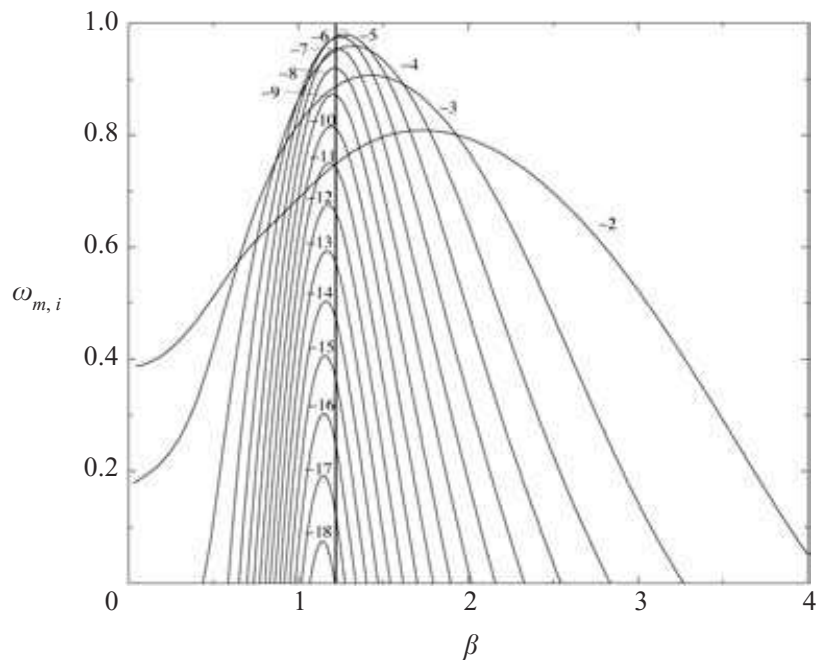

FIgURE 12. Model I growth rate $\omega_{m, i}$ as a function of helix angle $\beta=-k / m$ for $q=1.6$ and various values of $m$ in the range $-18 \leqslant m \leqslant-2$.

Leibovich \& Stewartson's (1983) analysis also predicts that the eigenfunctions are peaked in the vicinity of a critical radius $r_{c}$. For $q=1.2$, figure 11 depicts contours in the $(y, z)$-plane of the axial velocity eigenfunction pertaining to the $m=-5$ mode at $k=k_{-5}^{\max } \sim 4.9$, i.e. $\beta \sim 0.95$. The typical shape of a ring mode is obtained, which presents a sharp peak near $r_{-5}=1$ in close agreement with the theoretical prediction $r_{c}(q=1.2)=1$. Similar results hold when $q=1.6$ as seen in figure 12 . 

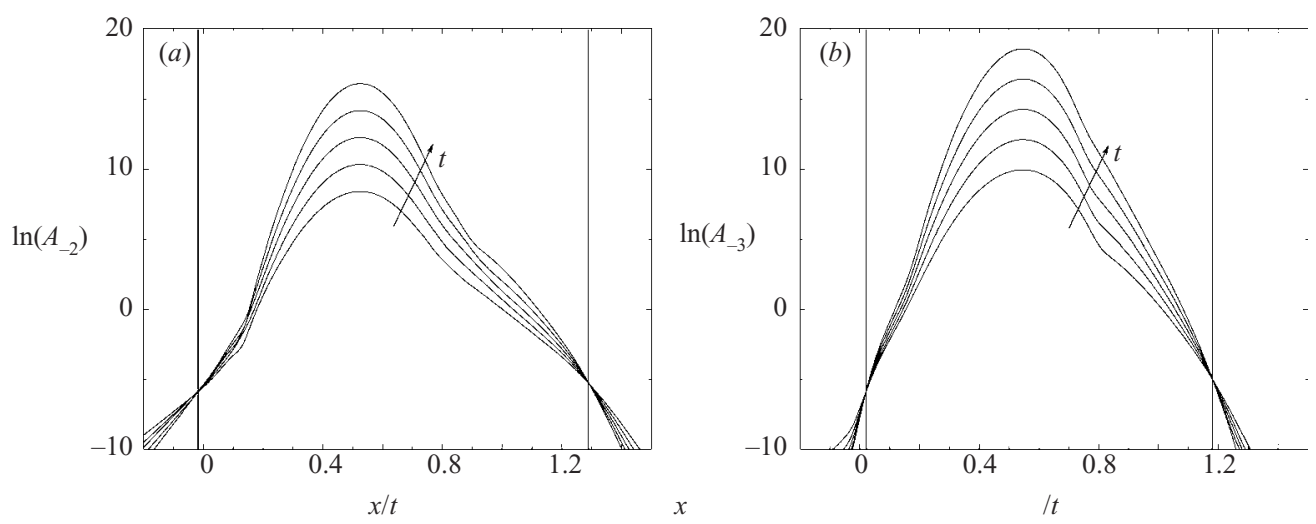

FIGURE 13. Spatio-temporal development of the impulse response wavepacket in model I for a swirl parameter $q=1.2$ and $R e=667$. The amplitude $A_{m}\left(v_{g}, t\right)$ is plotted as a function of the group velocity $v_{g}=x / t$ on a semi-log plot at different times $t=18,21,24,27,30$. (a) $m=-2$; (b) $m=-3$. Note that the $m=-2$ mode is absolutely unstable.

\section{Absolute/convective instability}

\subsection{Spatio-temporal evolution of the wavepacket}

In a first step, the advection parameter is set to $a=0$ and we focus on the spatiotemporal evolution of the wavepacket pertaining to different azimuthal wavenumbers $m$. Two characteristic examples of the time evolution of the impulse response on spatio-temporal rays $x / t=v_{g}$ are depicted in figures $13(a)$ and $13(b)$ at a swirl setting $q=1.2$ for $m=-2$ and $m=-3$ respectively. The amplitudes $A_{m}\left(v_{g}, t\right)$ are represented as a function of the group velocity $v_{g}=x / t$ on a semi-log plot at different times. Note that we do not resort to the Hilbert transform, as explained in $\S 4$. Therefore the properties of the positive helical mode $m=+2$ (resp. $m=+3$ ) remain hidden by the more unstable negative helical mode $m=-2$ (resp. $m=-3$ ).

It is seen that the wavepacket only grows in a range $\left[v_{m}^{-} ; v_{m}^{+}\right]$, where $v_{m}^{-}$and $v_{m}^{+}$are the group velocity of the trailing and leading edges of the wavepacket respectively. The sign of the trailing-edge velocity is of fundamental importance. When it is positive, the instability is convective, i.e. unable to withstand the advection as in figure $13(b)$ for $m=-3$. When it is negative, the instability is absolute: the wavepacket grows in place and ultimately contaminates the entire medium as in figure 13(a) for $m=-2$.

The corresponding spatio-temporal growth rates $\sigma_{m}\left(v_{g}\right)$, as defined in table 3, are plotted in figure 14 for $q=1.2$ and all azimuthal wavenumbers in the range $-11 \leqslant m \leqslant 0$. At $q=1.2$, negative helical modes of order as high as $m=-17$ are also unstable but for clarity we deliberately restrict the study to modes in the aforementioned range. Each $\sigma_{m}\left(v_{g}\right)$ curve in figure 14 contains all the information characterizing the spatio-temporal growth of the wavepacket. Its extent is delimited by the rays $v_{m}^{-}$and $v_{m}^{+}$of zero growth rate.

As explained in Delbende et al. (1998), the top of the curve coincides with the maximum temporal growth rate and it is reached on a ray of group velocity

$$
v_{m}^{\max }=\frac{\mathrm{d} \omega_{m, r}}{\mathrm{~d} k}\left(k_{m}^{\max }\right) .
$$

For instance for the mode $m=-5$, the maximum growth rate is $\sigma_{-5}^{\max }=0.77$ in perfect agreement with the temporal growth rate $\omega_{-5, i}^{\max }=0.78$ in figure 10 . The corresponding 


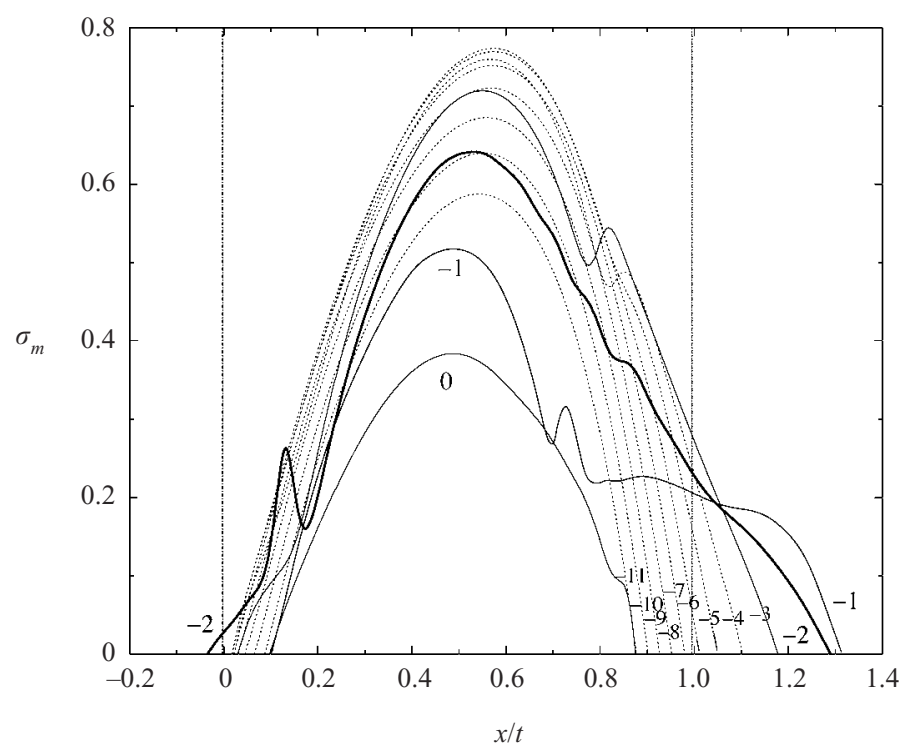

FIGURE 14. Spatio-temporal development of the wavepacket in model I for a swirl parameter $q=1.2$ and $R e=667$. The growth rate $\sigma_{m}\left(v_{g}\right)$ is represented as a function of the ray group velocity $v_{g}=x / t$ for values of $m$ ranging from $m=-11$ to $m=0$.

ray $v_{-5}^{\max }=0.57$ compares well with the group velocity calculated in the strictly temporal framework $\mathrm{d} \omega_{-5, r} / \mathrm{d} k\left(k_{-5}^{\max }\right)=0.55$. The bumps on figure 14 correspond to branch switchings.

It is customary to define the absolute growth rate according to

$$
\omega_{0, m, i} \equiv \sigma_{m}\left(v_{g}=0\right)
$$

i.e. it is the growth rate at zero group velocity. The sign of the absolute growth rate provides an alternative criterion to determine the nature of the instability. If negative, it points to a convective instability, if positive, to an absolute instability. There is only one absolutely unstable mode in figure 14: $m=-2$. The $m=-2$ mode is therefore expected to contaminate the entire medium, thereby giving birth to a global mode which will beat at the absolute frequency $\omega_{0,-2, r}$ and the absolute wavenumber $k_{0,-2, r}$. We have found that at incipient absolute instability $\left(\omega_{m, i}=0\right) k_{0,-2, r}=0.1$ and $\omega_{0,-2, r}=-0.35$ for $q=1.05$ and $R e=667$. These are the footprints of a negative helical mode rotating in time in the same direction as the underlying flow. These results are compared to the experimental observations of Billant et al. (1998) and Loiseleux \& Chomaz (2003) in $\S 7$. For reference, all curves $k_{r}(x / t)$ and $\omega_{r}(x / t)$ are given in figure 15 in the unstable range from $m=0$ to $m=-11$.

\subsection{Absolute/convective instability properties in $(a, q)$ parameter plane}

In absolute/convective instability analysis the free-stream velocity is known to play a crucial role. In the remainder of the study, the advection parameter $a$ is therefore varied. Since the absolute growth rate

$$
\omega_{0, m, i}(a, q) \equiv \sigma_{m}\left(v_{g}=0 ; a, q\right)
$$

entirely determines the convective or absolute nature of the instability, the curve $\omega_{0, m, i}(a, q)=0$ in the $(a, q)$ parameter plane is of fundamental importance in that 

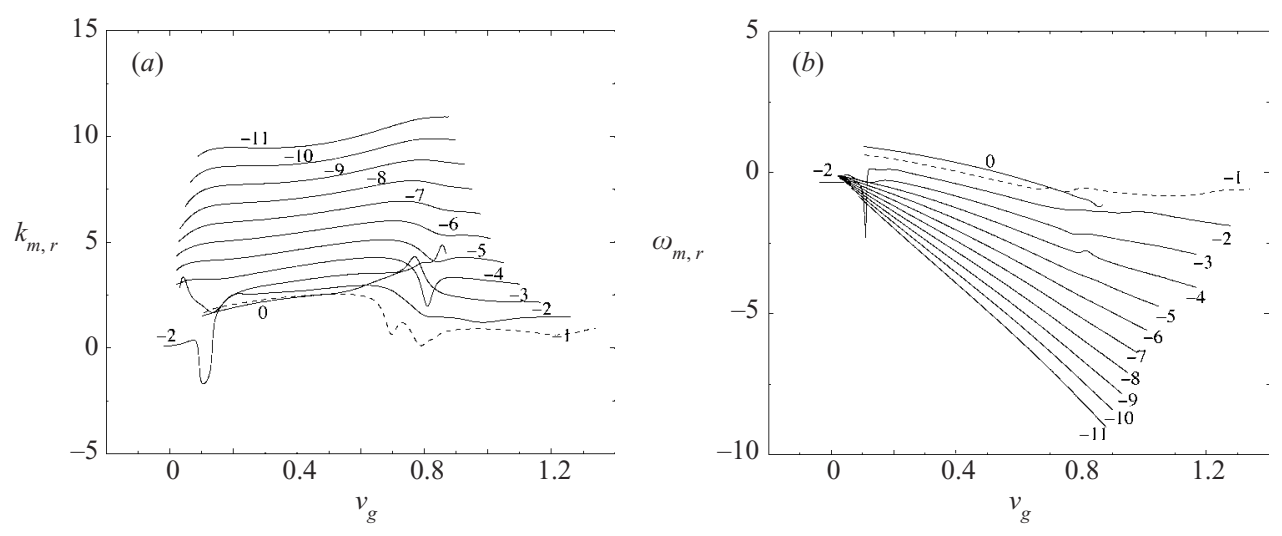

FIGURE 15. Distribution of $(a) k_{m, r}\left(v_{g}\right)$ and $(b) \omega_{m, r}\left(v_{g}\right)$ for model I at $a=0$, $q=1.2$ and $R e=667$.

it delineates the convective from the absolute instability domain. As pointed out in Delbende et al. (1998), one of the main advantages of the impulse response method is that one can deduce the absolute/convective nature of the instability for arbitrary values of the external flow parameter $a$ solely from a single numerical computation for zero external flow $(a=0)$. More precisely, Galilean invariance yields

$$
\omega_{0, m, i}(a, q)=\sigma_{m}\left(v_{g}=0 ; a, q\right)=\sigma_{m}\left(v_{g}=-a ; 0, q\right),
$$

which expresses that, at a given azimuthal wavenumber $m$ and swirl setting $q$, the boundaries $a_{m}^{-}(q)$ and $a_{m}^{+}(q)$ of the absolutely unstable domain are respectively the opposites of the velocities $v_{m}^{+}$and $v_{m}^{-}$of the leading and trailing edges of the wavepacket for zero free-stream velocity.

In order to determine the overall stability properties, the various absolute/ convective transition curves $\omega_{0, m, i}=0$ are overlaid in figure 16 for $m$ ranging from $m=-11$ to $m=0$. Curves corresponding to modes less than $m=-11$ are not included for clarity since they are all contained within the $m=-11$ curve and nested one inside the other. They do not therefore modify the outer CI/AI boundary. Open symbols refer to explicit calculation points whereas the minimum of each curve denoted by a solid circle has been extrapolated from the variations of the maximum spatio-temporal growth rate $\sigma_{m}^{\max }$ with the swirl parameter $q$.

For all azimuthal wavenumbers except $m=-2$, the absolutely unstable zone lies in the left half-plane $a<0$, which implies that counterflow is a prerequisite for absolute instability. As $q$ is increased, the extent of the AI range gradually widens for all unstable $m$. In contrast to the study of the Batchelor vortex, the modes do not restabilize at large swirl parameter values, due to the persistence of the azimuthal shear and centrifugal instabilities. But a stronger statement can be made: in the limit $q=\infty$, the base flow effectively reduces to a pure screened vortex without axial flow at infinite Reynolds number. The absence of axial flow and the unbroken symmetry $x \rightarrow \infty$ make it absolutely unstable. It can therefore be concluded that the region of absolute instability remains open for large $q$.

In the zero swirl case $(q=0)$, absolute instability for wakes $(a \leqslant-0.5)$ is due to the $m=-1$ mode (note that at $q=0$, the $m=+1$ and $m=-1$ modes have identical properties as discussed in §5) and only a small amount of counterflow $a>-0.98$ is necessary. This result is in excellent agreement with that of Monkewitz (1988), once 


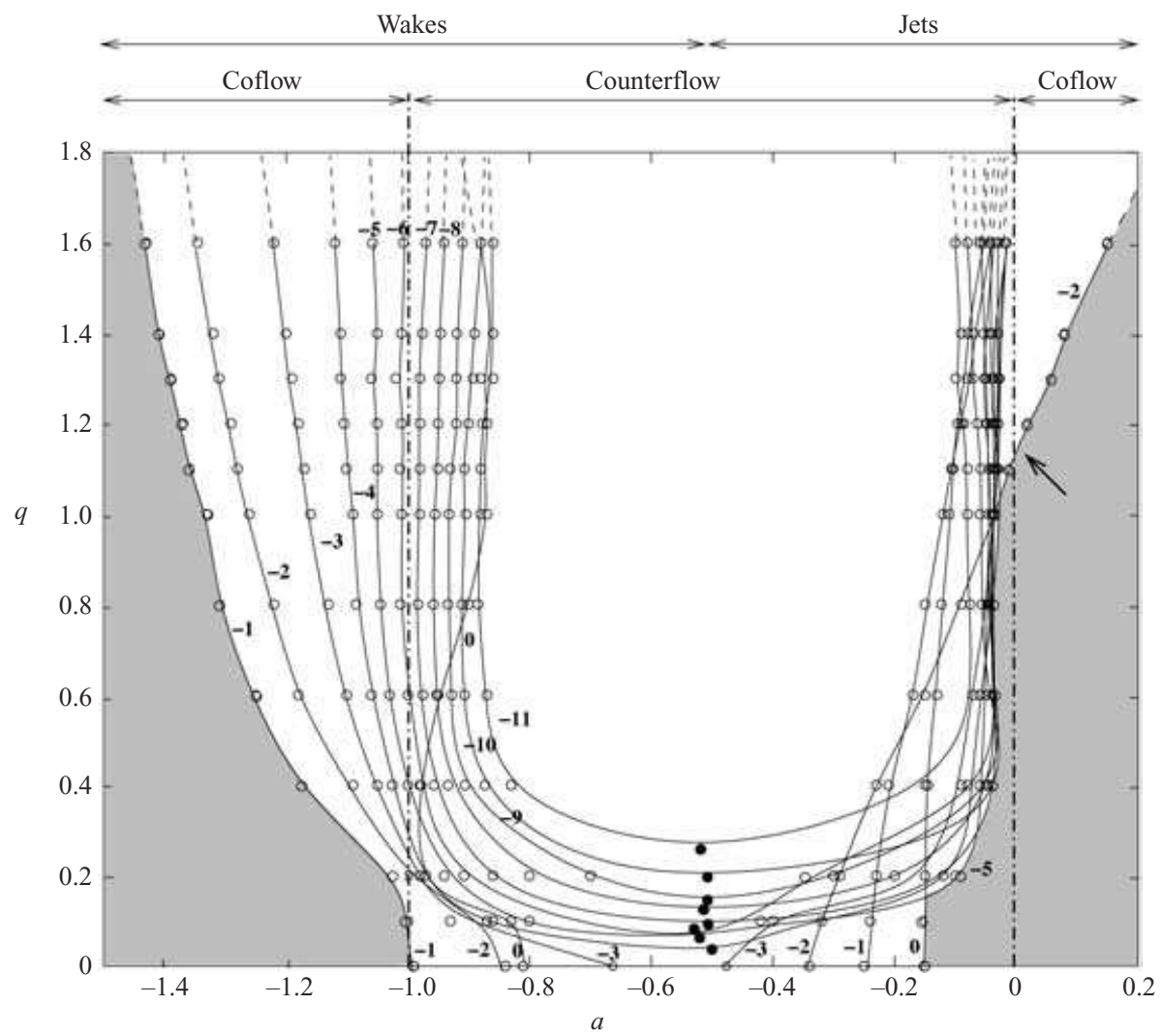

FIGURE 16. Regions of convective (shaded) and absolute (unshaded) instability in the $(a, q)$ parameter plane for the screened model I swirling jet profile at $R e=667$. Thin lines indicate $\mathrm{AI} / \mathrm{CI}$ transition curves for each azimuthal wavenumber $m$ ranging from $m=-11$ to $m=0$. Open symbols denote calculation points. Solid symbols have been extrapolated.

properly rescaled: according to his analysis, for $N=3$ at $R e=667$, which corresponds to a Reynolds number of 333 in Monkewitz' study, the wake is convectively unstable at $a=-1(\Lambda=-1)$ but absolutely unstable at $a=-0.95(\Lambda=-1.1)$. For jets $(a \geqslant-0.5)$, the axisymmetric mode $m=0$ is the first mode to become absolutely unstable at a counterflow $a=-0.15$. The instability of the zero coflow jet $(a=0)$ for $N=3$ is therefore convective at $R e=667$ in complete agreement with the result of Monkewitz $\&$ Sohn (1988) according to whom, for infinite Reynolds number and $N \rightarrow \infty$, the round jet becomes absolutely unstable for $a<-0.12(\Lambda=1.315)$.

As $q$ increases, the AI range widens and the critical transitional mode for absolute instability may change. For wakes, the transitional mode remains $m=-1$. Wakes without counterflow $(a=-1)$ undergo a transition to AI for $q_{c}=0.08$. This is analogous to the Batchelor vortex, for which the transition takes place at $q=0.13$ (see Delbende et al. 1998) for the mode $m=-1$. The predominance of the $m=-1$ mode seems to be a very robust feature of most swirling wake models studied in the literature.

In the case of jets with counterflow $(-0.5<a<0)$, the transitional mode stays at $m=0$ for small $q$ but it is soon replaced by $m=-5$. As $q$ is further increased, the transitional mode varies from $m=-6$ to $m=-7$ until, for a swirl value of $q=1$, the 

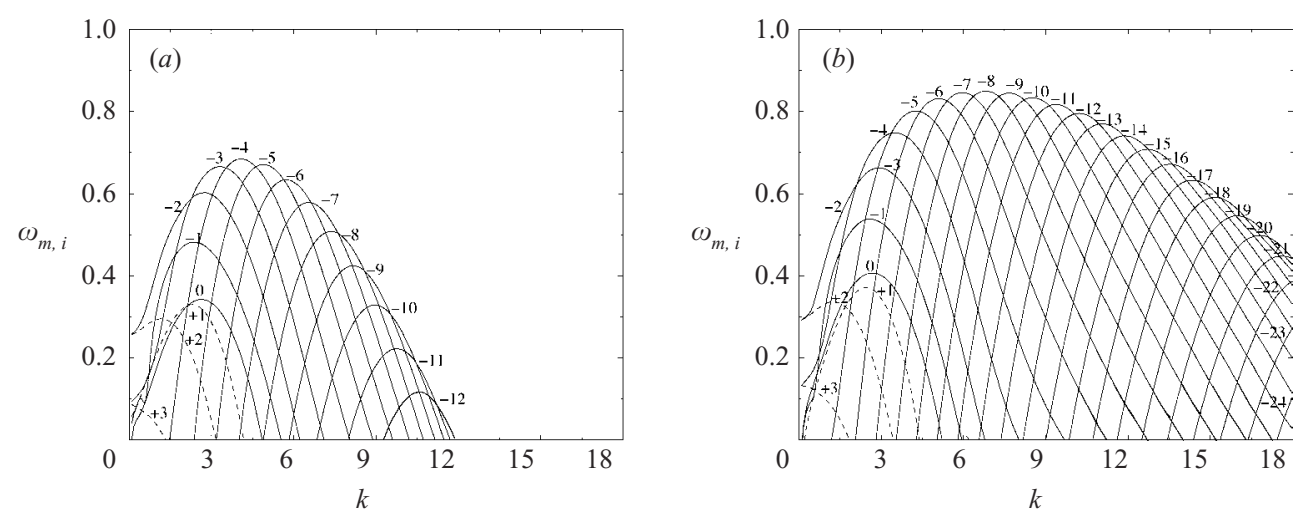

Figure 17. Effect of the Reynolds number on Model I temporal instability at $q=1.2$. The growth rate $\omega_{m, i}(k)$ is plotted as a function of axial wavenumber $k$. (a) $R e=333 ;(b) R e=1333$; compare also with figure 7.

$m=-2$ mode becomes the 'most absolute'. At $q=1.05, m=-2$ becomes absolutely unstable at $a=0$ and it is above this critical swirl setting that jets with coflow become absolutely unstable. As $q$ continues to increase, the $m=-2$ mode becomes more strongly absolute, even in the presence of coflow.

In contrast to the Batchelor vortex (Delbende et al. 1998; Olendraru et al. 1999; Olendraru \& Sellier 2002), the model I screened swirling jet presents a well-defined transition from convective to absolute instability as the swirl parameter is increased above $q=1.05$ through the mode $m=-2$. Higher-order modes $(m=-3,-4 \ldots)$ all present the same behaviour with a transition on the jet side taking place close to $a=-0.03$ in the range $0.6 \leqslant q \leqslant 1.6$.

In the case of the Rankine vortex with plug axial flow (Loiseleux et al. 1998), absolute/convective instability transition curves in the $(a, q)$ parameter plane and pertaining to different negative azimuthal wavenumbers are closed on the high- $q$ side. However, the overall absolutely unstable domain remains open for large $q$, since azimuthal modes of arbitrarily high order remain unstable in the absence of a shear-layer length scale.

\section{Effect of the Reynolds number}

As argued in $\S 5.6$, modes with arbitrary large wavenumbers and the appropriate $\mathrm{k} / \mathrm{m}$ ratio have the same growth rate as predicted by the inviscid asymptotic theory of Leibovich \& Stewartson (1983) but they are expected to be quenched in the presence of a finite viscosity. As a complement to figure 7(a), figure 17 depicts the temporal instability curves of model I and a swirl setting $q=1.2$ for two other Reynolds numbers $R e=333$ and $R e=1333$. At $R e=333$ (figure 17a), modes with $m<-12$ are stabilized and the most unstable mode is found to be $m=-4$. In contrast, modes as high as $m=-27$ (out of the plotted range) are unstable at $R e=1333$ and the most unstable mode is $m=-8$ (figure $17 b$ ).

The influence of viscosity is further analysed in figure 18 where the maximum growth rate $\sigma_{m}^{\max }$ that occurs at a value of $k$ denoted $k_{m}^{\max }$ (of order $m \beta_{\max }$ ) for all unstable $m \leqslant-2$ is plotted as a function of the square of the total wavenumber $k_{t o t}^{2}=m^{2}+k_{m}^{m a x}{ }^{2}$ at $q=1.2$ and for three different Reynolds numbers. Figure 18 show 


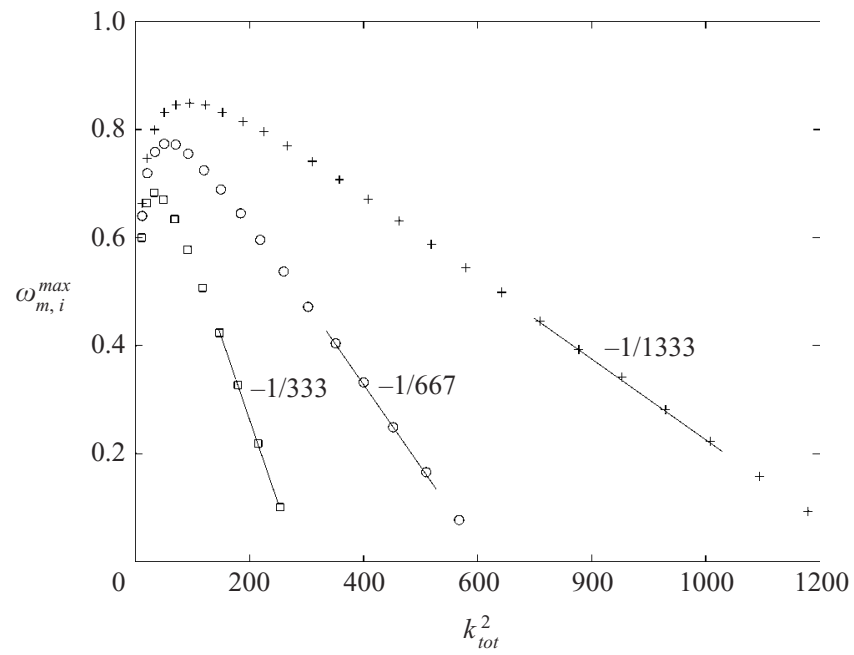

FIGURE 18. Model I maximum growth rates $\omega_{m, i}^{\max }$ corresponding to all unstable azimuthal wavenumbers $m \leqslant-2$ as a function of the square of the total wavenumber $k_{t o t}^{2}=m^{2}+k_{m}^{m a x^{2}}$ for $q=1.2$ for three different Reynolds numbers: $R e=333(\square), R e=667(\bigcirc)$ and $R e=1333(+)$; $-1 / \operatorname{Re}$ slopes are also displayed.

\begin{tabular}{rcccccccccc}
\hline (a) $R e \backslash m$ & 0 & -1 & -2 & -5 & (b) $R e \backslash m$ & 0 & -1 & -2 & -5 \\
333 & -0.86 & -1.33 & -1.27 & -1.02 & 333 & -0.10 & -1.09 & -0.00 & -0.03 \\
667 & -0.87 & -1.38 & -1.29 & -1.05 & 667 & -0.09 & -0.09 & +0.02 & -0.03 \\
1333 & -0.89 & -1.40 & -1.30 & -1.07 & 1333 & -0.09 & -0.09 & +0.05 & -0.03
\end{tabular}

TABLE 4. Influence of the Reynolds number on the wavepacket edges at $q=1.2$, model I for several azimuthal modes $m$. (a) Amount of minimal coflow parameter $a$ to trigger AI for wakes. (b) Amount of minimal coflow parameter $a$ to trigger AI for jets.

that the growth rates decrease in proportion to $-k_{t o t}^{2} / R e$. This scaling law may be explained by the balance between the inviscid asymptotic growth rate (5.3) and the viscous dissipation. Since the eigenmode is assumed to vary slowly in the radial direction the dissipation may be estimated from the bulk contribution of the viscous term $(1 / R e) \nabla^{2} \sim(1 / R e)\left(k^{2}+m^{2}\right)$.

Regarding spatio-temporal stability, the analysis of Olendraru \& Sellier (2002) on the Batchelor vortex has shown that increasing the Reynolds number tends to only slightly increase the size of the AI range, once the Reynolds number is larger than a few hundred. The same trend is found here and the differences for all azimuthal modes and Reynolds numbers $R e=333,667$ and 1333 are found to remain extremely small. For this reason the critical advection parameters corresponding to AI/CI transition are now given only for a few modes $m=0,-1,-2$ and -5 .

The necessary amount of counterflow $a$ to trigger absolute instability is listed in table 4 for some representative helical modes $m=0,-1,-2$ and -5 and for the same swirl setting as previously, $q=1.2$. Table $4(a)$ refers the wakes $(a<-0.5)$ whereas table $4(b)$ refers to jets $(a>-0.5)$.

The main obervations of Olendraru \& Sellier (2002) are confirmed for the present screened swirling jet model I: the extent of the absolutely unstable $a$-range is enlarged 

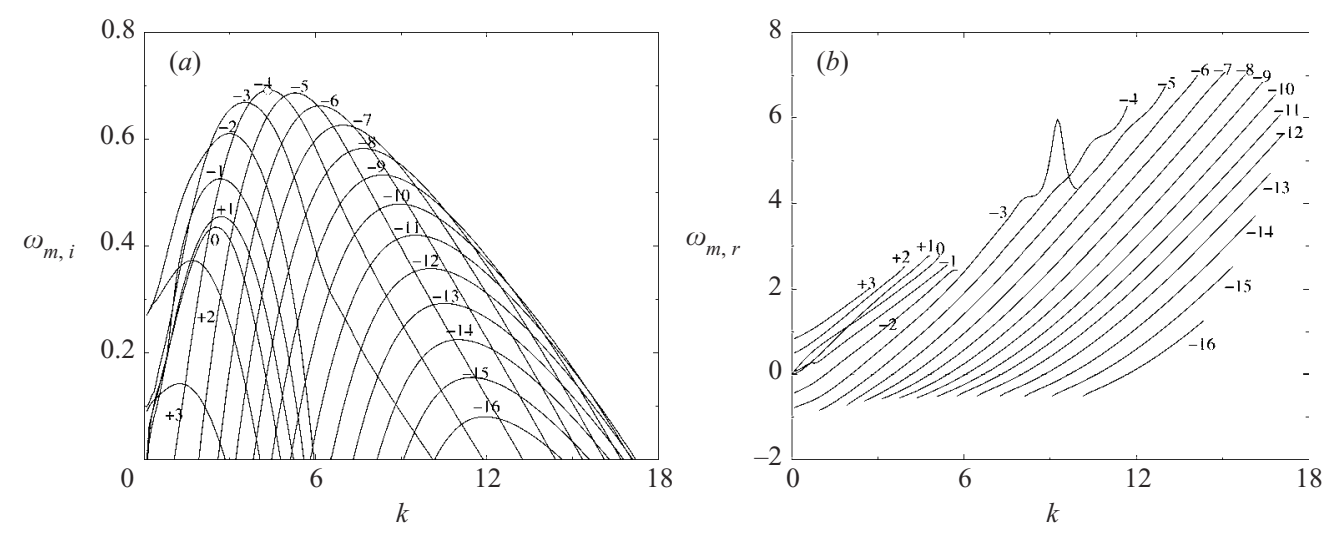

FIGURE 19. Model II temporal instability at $q=1.16:(a)$ growth rate $\omega_{m, i}(k)$ as a function of axial wavenumber $k ;(b)$ frequency $\omega_{m, r}(k)$ as a function of $k$.

as the Reynolds number is increased. On the wakeside $(a<-0.5)$, the threshold $a$ values decrease with $R e$ whereas they increase on the jet side. All these variations are however very weak, i.e. less than a few percent of the typical velocity $U_{c}-U_{\infty}$. The results obtained for $R e=667$ in the previous section are therefore generic and apply with minor modifications to other Reynolds numbers.

\section{Mode selection: comparison with experiment}

We have performed three impulse response simulations for the model II base flow (2.5) with an axial velocity spike at $q=0.82(S=0.68), q=1.16(S=0.92)$ and $q=1.48$ $(S=1.08)$. As illustrated in figure 19 at $q=1.16$, the temporal stability results are found to be only weakly affected in comparison to the model I flow $(2.3 a-c)$ without a spike (see figure 7 at $q=1.2$ ). Note that the growth rate envelopes are qualitatively similar but that the most unstable azimuthal mode may differ: $m=-4$ for model II and $m=-5$ for model I.

The corresponding spatio-temporal analysis for these three model II cases is summarized in figure 20 which only displays the transition curves of three representative helical modes. On the wake side $(a \leqslant-0.5)$, the $m=-1$ mode is critical for both models, the transition curve of model II being slightly shifted towards the coflow region to the left. On the jet side $(a \geqslant-0.5)$, the $m=-1$ transition curves are identical for both models. For $m=-2$ the absolute domain is slighty narrower and the transition for zero coflow jets $(a=0)$ is shifted a few percent towards higher $q$. Still, both models behave very similarly and only mode $m=-2$ becomes absolutely unstable in the absence of counterflow $(a \geqslant 0)$. Finally, the absolutely unstable domain of mode $m=-5$ is almost unaffected on both the wake and jet sides. It may therefore be concluded that the simpler model I flow does indeed capture the dominant spatio-temporal features of the instability. The axial velocity overshoot is seen to have a negligible impact. Model I has the advantage that it decouples the vortex from the jet through a single parameter $q$ which proves to provide a very good approximation to the instability characteristics of the more intricate experimental flow.

We now proceed to compare the spatio-temporal properties pertaining to model II with the pre-breakdown dynamics in swirling jets observed by Billant et al. 


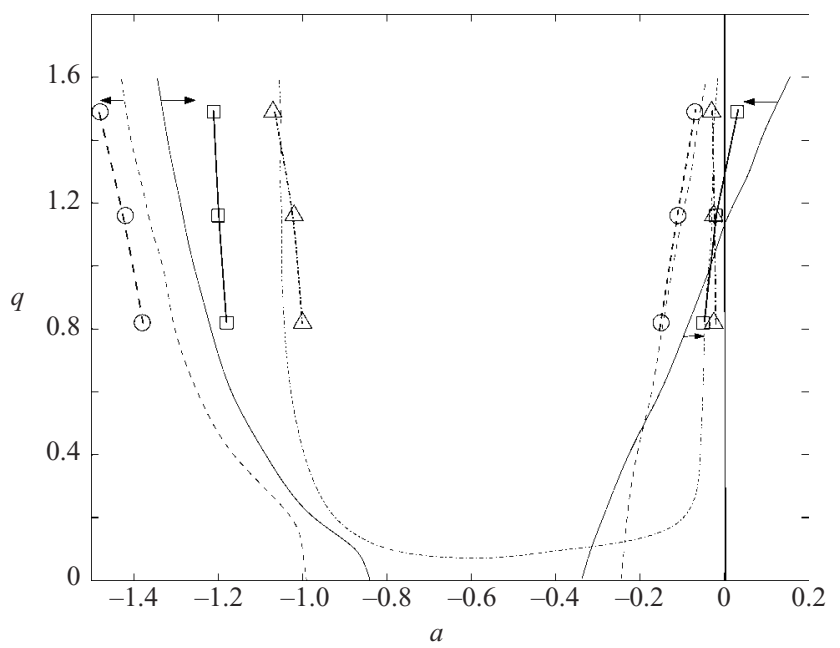

FiguRE 20. Convective/absolute instability transition curves $m=-1(\bigcirc),-2(\square),-5(\triangle)$ for model II screened swirling jet at $R e=667$ (lines with symbols). For comparison, corresponding curves from figure 16 are also displayed for model I (lines without symbols).

(1998). According to figure 16 for model I, zero coflow jets $(a=0)$ undergo a convective/absolute instability transition for the critical swirl level $q=1.05$, for which the absolute wavenumber is found to be $k_{0,-2, r}=0.1$ and the real absolute frequency is $\omega_{0,-2}=\omega_{0,-2, r}=-0.35$. Provided one assumes the unsteady pre-breakdown state to be triggered and sustained by a front at the convective/absolute instability location (see the introduction for a discussion), then it inherits $\omega_{0,-2}$ and $k_{0,-2, r}$ as its frequency and wavenumber respectively. The analysis then predicts that the unsteady structure takes the form of a negative $m=-2$ helical mode wih a pitch $2 \pi / k_{0,-2, r} \times 1 /|m|=31$ radii and a period $q /\left|\omega_{0,-2}\right|=3.3$ times the rotation period of the swirling base flow. Furthermore, following the discussion in $\S 3$, this helical mode rotates in the same direction as the underlying swirling jet. In the experiment of Billant et al. (1998), for $q=1.3$ and $R e=666$, the pre-breakdown structure consists of a double helix winding in the same direction as the background rotation $\dagger$ with a pitch of the order of 8 jet radii. With regard to the temporal variations, we note that Billant et al. (1998) fail to detect any time evolution of the double helix while Loiseleux \& Chomaz (2003) report that at $R e=1490$ and $S=0.86$ the helix rotates extremely slowly in time in the same direction as the swirl with a period close to 3 times the background rotation period, in good qualitative agreement with the present theoretical results $(3.3$ times). As discussed in the introduction, real swirling jets are non-parallel. In such cases, predictions of the global mode characteristics based solely on local absolute properties (in the present study at a station close to the inlet) are only qualitative since non-parallel effects are no longer negligible and may modify the growth rate and

$\dagger$ It should be emphasized that the flow rotation around the jet axis in the experiment of Billant et al. (1998) is considered to be positive according to a left-hand rule with respect to the jet direction. In order to follow the convention adopted in the present study it should therefore be regarded as negative. In view of the symmetry relation discussed in $\S 3$, their $m=+2$ mode is an $m=-2$ mode according to the right-hand rule. Independently of the convention chosen, it winds in the same direction as the underlying flow rotation. 
the frequency, although they are not expected to affect the azimuthal mode selection. An estimate of these second-order effects would require the precise knowledge of the basic flow, which is not available from the experiment since nonlinear transfers are present further downstream due to the saturation of the instability.

\section{Conclusions and discussion}

The main result of the study concerns mode selection in the pre-breakdown stage of swirling jets dynamics. According to $\S \S 6$ and 7, the swirling jet selects as a self-sustained mode a double helix $m=-2$ helical mode which becomes absolutely unstable above the critical swirl level $q=1.05$. A temporal analysis alone is incapable of selecting such a mode. It predicts that $m=-5$ exhibits the largest temporal growth rate, a mode which is not observed experimentally. It is the absolutely unstable nature of $m=-2$ which allows its emergence from the background noise. This finding is based on the spatio-temporal instability properties of the velocity profiles close to the nozzle exit. It implies that this station acts as a wavemaker for the entire flow. It remains to be established that a nonlinear global mode may indeed exist on the spatially developing swirling jet, with a front located at the inlet, in the same spirit as the theoretical investigations of Couairon \& Chomaz (1999). Because of the non-parallelism of the flow, second-order effects are not negligible and the agreement between theory and experiment remains somewhat qualitative: though the azimuthal symmetry of the dominant mode is precisely predicted and the frequency of the double helix as well as the threshold swirl value are in the right range, the wavenumber prediction is poor however. This is not only a consequence of nonparallel effects; it is known from the theory that the wavenumber is determined by nonlinear effects and therefore intrisically varies along the stream.

A recent theoretical investigation by Yin et al. (2000) has demonstrated that the absolute instability of the Batchelor vortex with a wake $(a \leqslant-0.5)$ succeeds in accounting for the $m=-1$ helical structure experimentally observed downstream of vortex breakdown by Garg \& Leibovich (1979). In particular, the dominant Strouhal number in the wake of vortex breakdown is correctly predicted as well as the winding in the opposite direction to the underlying swirling motion (see $\S 3$ for a justification of this last property). All existing studies reveal that the most absolutely unstable mode in swirling wakes is $m=-1$, whatever basic profiles are used. It is a robust feature of swirling wake dynamics. However, double helices $(m=-2)$ have also been observed for sufficiently high swirl in the lee of the breakdown bubble in recent three-dimensional direct numerical simulations of vortex breakdown in a semi-infinite domain by Ruith \& Meiburg (2002).

All these conclusions imply that absolute/convective instablity concepts appear to be successful in predicting the unsteady structure of swirling flows, whether they are in a pre-breakdown stage $(m=-2)$ or in full breakdown configuration $(m=-1)$. They fail, however, to explain the onset of vortex breakdown, which does not appear to a rise from a local instability but rather from an overall modification of the basic flow.

The temporal instability analysis has revealed that a large range of negative helical modes (winding with the underlying swirl) become and remain unstable as swirl is increased. In the specific case of screened swirling jets, three instability mechanisms have been identified which are associated with axial shear, azimuthal shear and centrifugal forces. It is the latter, in its generalized form for helical disturbances in swirling jets, which appears to explain the temporal mode selection. Furthermore, the instability characteristics (growth rate, wavenumbers 
and eigenfunction) at large azimuthal wavenumber $|m|$ have been shown to agree remarkably well with the analytical predictions of Leibovich \& Stewartson (1983). This generalized instability mechanism is active for all continuous velocity profiles and its comparison with the Batchelor model explains why the temporal characteristics of the instability at moderate values of the swirl parameter are similar for both models despite their differences. This concerns only temporal instability however and not absolute/convective transition that involves lower azimuthal modes and other mechanisms, in which the screening effects seem essential and the use of realistic velocity profiles mandatory. In closing, it is worth emphasizing that the double helix configuration is observed not only in the screened swirling jet experiments of Billant et al. (1998) upon which the present study is based, but also in swirling flows through confined tubes (Sarpkaya 1971; Escudier et al. 1980).

I. Delbende and P. Billant are warmly acknowledged for stimulating discussions and P. Huerre for helpful suggestions and constant encouragement. The work is supported by the French Ministry of Defence under grant No. 99001004707588 and by IDRIS (CNRS) for computational facilities under project No. 020174.

\section{REFERENCES}

Ash, R. L. \& Khorrami, M. R. 1995 Vortex stability. In Fluid Vortices (ed. S. I. Green), chap. 8, pp. 317-372. Kluwer.

BAtchelor, G. K. \& Gill, A. 1964 Analysis of the stability of axisymmetric jets. J. Fluid Mech. 14, 529-551.

Benjamin, T. B. 1962 Theory of the vortex breakdown phenomenon. J. Fluid Mech. 14, 593-629.

BERS, A. 1983 Space-time evolution of plasma instabilities-absolute and convective. In Handbook of Plasma Physics (ed. M. Rosenbluth \& R. Sagdeev), pp. 451-517. North-Holland.

Billant, P., Chomaz, J.-M. \& Huerre, P. 1998 Experimental study of vortex breakdown in swirling jets. J. Fluid Mech. 376, 183-219.

Brancher, P. \& Chomaz, J.-M. 1997 Absolute and convective instabilities in spatially periodic shear flows. Phys. Rev. Lett. 78, 658-661.

Brancher, P., Chomaz, J.-M. \& Huerre, P. 1994 Direct numerical simulations of round jets: vortex induction and side-jets. Phys. Fluids 6, 1768-1774.

Carnevale, G. \& Kloosterziel, R. 1994 Emergence and evolution of triangular vortices. J. Fluid Mech. 259, 305-331.

Carton, X. \& McWilliams, J. 1989 Barotropic and baroclinic instabilties of axisymmetric vortices in a quasi-geostrophic model. In Mesoscale/Synoptic Coherent Structures in Geophysical Turbulence (ed. J. Nihoul \& B. Jamart), pp. 225-244. Elsevier.

Сhomaz, J.-M. 1992 Absolute and convective instability in non linear systems. Phys. Rev. Lett. 69, 1931-1934.

Couniron, A. \& Chomaz, J.-M. 1997 Absolute and convective instabilities, front velocities and global modes in nonlinear systems. Physica D 108, 236-276.

Couairon, A. \& Chomaz, J.-M. 1999 Fully nonlinear global modes in slowly varying flows. Phys. Fluids 11, 3688-3703.

Delbende, I., Chomaz, J.-M. \& Huerre, P. 1998 Absolute/convective instabilities in the Batchelor vortex: a numerical study of the linear impulse response. J. Fluid Mech. 355, 229-254.

Delerr, J. 1994 Aspects of vortex breakdown. Prog. Aerospace. Sci. 30, 1-59.

Drazin, P. \& ReID, W. 1981 Hydrodynamic Stability. Cambridge University Press.

Duck, P. \& Foster, M. 1980 The inviscid stability of a trailing line vortex. Z. Angew Math. Phys. 31, 524-532.

Emanuel, K. 1984 A note on the stability of columnar vortices. J. Fluid Mech. 145, 235-238.

Escudier, M., Bornstein, J. \& Maxworthy, T. 1982 The dynamics of confined vortices. Proc. R. Soc. Lond. A 382, 335-360. 
EsCudier, M., Bornstein, J. \& ZEHNDER, N. 1980 Observation and LDA measurements of confined turbulent vortices. J. Fluid Mech. 98, 49-63.

Faler, J. \& Leibovich, S. 1977 Disrupted states of vortex flow and vortex breakdown. Phys. Fluids 20, $1385-1400$.

Gallaire, F. \& Chomaz, J.-M. 2003 Three-dimensional instability of isolated vortices. Phys. Fluids 15, 2113-2126.

Garg, A. \& Leibovich, S. 1979 Spectral characteristics of vortex breakdown flowfields. Phys. Fluids 22, 2053-2064.

Gent, P. \& McWilliams, J. 1986 The instability of barotropic circular vortices. Geophys. Astrophys. Fluid Dyn. 35, 209-233.

HowARD, L. \& GuPTA, A. 1962 On the hydrodynamic and hydromagnetic stability of swirling flows. J. Fluid Mech. 14, 463-476.

Huerre, P. 2000 Open shear flow instabilities. In Perpectives in Fluid Dynamics (ed. G. K. Batchelor, H. K. Moffatt \& M. G. Worster), pp. 159-229. Cambridge University Press.

Huerre, P. \& Rossi, M. 1998 Hydrodynamic instabilities in open flows. In Hydrodynamic and Nonlinear Instabilities (ed. C. Godrèche \& P. Manneville), pp. 81-294. Cambridge University Press.

Khorrami, M. R. 1991 On the viscous modes of instability of a trailing line vortex. J. Fluid Mech. 225, 197-212.

LeIbovich, S. 1984 Vortex stability and breakdown: survey and extension. AIAA J. 22, 1192-1206.

Leibovich, S. \& Stewartson, K. 1983 A sufficient condition for the instability of columnar vortices. J. Fluid Mech. 126, 335-356.

Lessen, M. \& Paillet, F. 1974 The stability of a trailing line vortex. Part 2. Viscous theory. J. Fluid Mech. 65, 769-779.

Lessen, M. \& Singh, P. J. 1974 The stability of axisymmetric free shear layers. J. Fluid Mech. 60, 433-457.

Lessen, M., Singh, P. J. \& Paillet, F. 1974 The stability of a trailing line vortex. Part 1. Inviscid theory. J. Fluid Mech. 63, 753-763.

Liang, H. 2003 Experimental investigations of swirling jets. PhD thesis, University of Southern California.

Lim, D. \& Redekopp, L. 1998 Absolute instability conditions for variable density, swirling jet flows. Eur. J. Mech. B/Fluids 17, 165.

Loiseleux, T. \& Chomaz, J.-M. 2003 Breaking of rotationnal symmetry in a swirling jet experiment. Phys. Fluids 15, 511-523.

Loiseleux, T., Chomaz, J.-M. \& Huerre, P. 1998 The effect of swirl on jets and wakes: Linear instability of the Rankine vortex with axial flow. Phys. Fluids 10, 1120:1134.

Ludwieg, H. 1960 Stabilität der Strömung in einem zylindrischen Ringraum. Z. Flugwiss. 8, 135-142.

Ludwieg, H. 1961 Ergänzung zu der Arbeit: "Stabilität der Strömung in einem zylindrischen Ringraum". Z. Flugwiss. 9, 359-361.

Mayer, E. \& Powell, K. 1992 Viscous and inviscid instabilities of a trailing line vortex. J. Fluid Mech. 245, 91-114.

Michalke, A. 1984 Survey on jet instability theory. Prog. Aerospace Sci. 21, 159-199.

Monkewitz, P. 1988 A note on vortex shedding form axisymmetric bluff bodies. J. Fluid Mech. 192, $561-575$.

Monkewitz, P. \& Sohn, K. 1988 Absolute instability in hot jets. AIAA J. 26, 911-916.

Olendraru, C. \& Sellier, A. 2002 Viscous effects in the absolute-convective instability of the Batchelor vortex. J. Fluid Mech. 459, 371-396.

Olendraru, C., Sellier, A. \& Huerre, P. 1999 Inviscid instability of the Batchelor vortex: Absolute/convective transition and spatial branches. Phys. Fluids 11, 1805-1820.

Orlandi, P. \& Carnevale, G. 1999 Evolution of isolated vortices in a rotating fluid of finite depth. J. Fluid Mech. 381, 239-269.

Pier, B. \& Huerre, P. 2001 Nonlinear self-sustained structures and fronts in spatially developing wake flows. J. Fluid Mech. 435, 145-174.

RAYLEIGH, LORD 1892 On the instability of cylindrical fluid surfaces. Phil. Mag. 34, 177-180.

RaYleigh, Lord 1916 On the dynamics of revolving fluids. Proc. R. Soc. Lond. A 93, 148-154. 
Ruith, M. \& Meiburg, E. 2002 Direct numerical simulation of spatially developing, threedimensional swirling jets. J. Turbulence 3, art. no. 065 .

SARPKAYA, T. 1971 On stationary and travelling vortex breakdown. J. Fluid Mech. 45, 545-559.

Schwartz, M., Bennett, W. \& Stein, S. 1966 Communication Systems and Techniques. McGraw-Hill. Spall, R. E., Gatski, T. B. \& Grosch, C. 1987 A criterion for vortex breakdown. Phys. Fluids 30, 3434-3440.

Stewartson, K. \& Leibovich, S. 1987 On the stability of a columnar vortex to disturbances with large azimuthal wavenumber: the lower neutral points. J. Fluid Mech. 178, 549-566.

Synge, J. L. 1933 The stability of heterogeneous liquids. Trans. R. Soc. Can. 27, 1-18.

Yin, X., Sun, D., WeI, M. \& Wu, J. 2000 Absolute and convective instablity character of slender viscous vortices. Phys. Fluids 12, 1062-1072. 